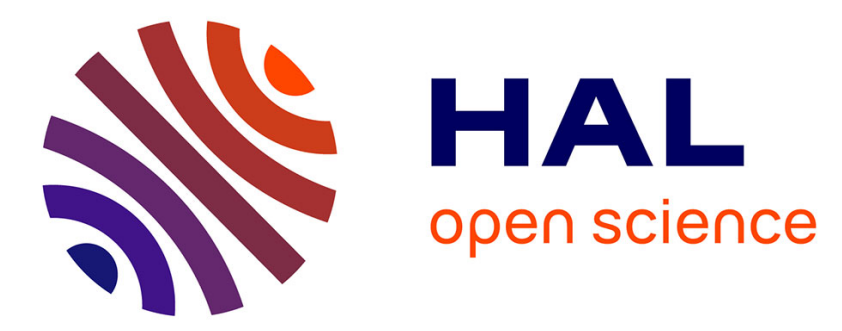

\title{
Universality of the optical absorptance quantization in two-dimensional group-IV, III-V, II-VI and IV-VI semiconductors
}

Michel Lannoo, P Tim Prins, Zeger Hens, Daniel Vanmaekelbergh, Christophe Delerue

\section{To cite this version:}

Michel Lannoo, P Tim Prins, Zeger Hens, Daniel Vanmaekelbergh, Christophe Delerue. Universality of the optical absorptance quantization in two-dimensional group-IV, III-V, II-VI and IV-VI semiconductors. Physical Review B, 2022, 105, pp.035421. 10.1103/PhysRevB.105.035421 . hal-03536349

\section{HAL Id: hal-03536349 https://hal.science/hal-03536349}

Submitted on 19 Jan 2022

HAL is a multi-disciplinary open access archive for the deposit and dissemination of scientific research documents, whether they are published or not. The documents may come from teaching and research institutions in France or abroad, or from public or private research centers.
L'archive ouverte pluridisciplinaire $\mathbf{H A L}$, est destinée au dépôt et à la diffusion de documents scientifiques de niveau recherche, publiés ou non, émanant des établissements d'enseignement et de recherche français ou étrangers, des laboratoires publics ou privés. 


\title{
Universality of the optical absorptance quantization in two-dimensional group-IV, III-V, II-VI and IV-VI semiconductors
}

\author{
Michel Lannoo, ${ }^{1}$ P. Tim Prins,${ }^{2}$ Zeger Hens,${ }^{3}$ Daniel Vanmaekelbergh,${ }^{2}$ and Christophe Delerue ${ }^{4} *$ \\ ${ }^{1}$ Aix Marseille Université, CNRS, Université de Toulon, IM2NP UMR 7334, 13397, Marseille, France \\ ${ }^{2}$ Debye Institute for Nanomaterials Science, University of Utrecht, \\ Princetonplein 1, 3584 CC Utrecht, The Netherlands \\ ${ }^{3}$ Physics and Chemistry of Nanostructures (PCN), Ghent University, 9000 Gent, Belgium \\ ${ }^{4}$ Université de Lille, CNRS, Centrale Lille, Université Polytechnique Hauts-de-France, \\ Junia, UMR 8520 - IEMN, F-59000 Lille, France
}

\begin{abstract}
The optical absorptance of a single graphene layer over a wide range of wavelengths is known to be remarkably constant at the universal value $\pi \alpha$ where $\alpha$ is the fine structure constant. Using atomistic tight-binding calculations, we show that the absorptance spectra of nanometer-thin layers (quantum wells) of group-IV, III-V, II-VI or IV-VI semiconductors are characterized by marked plateaus at integer values of $\pi \alpha$, in the absence of excitonic effects. In the case of InAs, the results obtained are in excellent agreement with the currently available experimental data. By revisiting experimental data on semiconductor superlattices, we show that $\pi \alpha$ is also a metric of their absorption when normalized to a single period. We conclude that the $\pi \alpha$ quantization is universal in semiconductor quantum wells provided that excitonic effects are weak, and is therefore not specific to the zero-gap graphene case. The physical origin of this universality and its limits are discussed using analytical models that capture the main underlying physics of the lowest optical transitions in III-V and II-VI semiconductor quantum wells. These models show that the absorptance is ruled by $\pi \alpha$ independent of the material characteristics because of the presence of a dominant term in the Hamiltonian, linear in the wave vector $\mathbf{k}(\sim \mathbf{V} \cdot \mathbf{k})$, which couples the conduction band to the valence bands. However, the prefactor in front of $\pi \alpha$ is not unity as in graphene due to the different nature of the electronic states. In particular, the spin-orbit coupling plays an important role in bringing the absorptance plateaus closer to integer values of $\pi \alpha$. The case of IV-VI semiconductor ( $\mathrm{PbSe}$ ) quantum wells characterized by a rocksalt lattice and multi-valley physics is very similar to that of graphene, with the specification that a "massful gap" is formed around the Dirac points.
\end{abstract}

\section{INTRODUCTION}

The fine structure constant $\alpha$, introduced by Arnold Sommerfeld [1], characterizes the strength of the interactions between charged particles and the electromagnetic field [2]. In condensed matter physics, this constant quantifies the response to electromagnetic excitation of a large number of very different systems, such as materials with topological phases [3] including the quantum Hall effect [4], graphene [5-10], black phosphorus multilayers [11] or plasmonic networks [12]. In particular, if an undoped graphene monolayer is irradiated, the amount of absorbed light in a wide range of wavelengths is given by the universal constant $\pi \alpha \approx 2.3 \%$. Remarkably, this same value appears in the quantization of optical absorption through InAs nanometer thick layers (hereinafter referred to as quantum wells) [13]. In this case, after correction of local-field effects, the absorptance spectrum is characterized by a succession of several plateaus whose respective heights are very close to $\pi \alpha$. Surprisingly, however, the case of quantum wells of semiconductors other than InAs has not been studied so far.

This quantization is not universal. For example, ultrafine monolayers obtained by exfoliation of van der Waals

\footnotetext{
* christophe.delerue@iemn.fr
}

materials, such as transition metal dichalcogenides, often show spectra of great richness, with bound exciton absorptances much higher than $\pi \alpha$, especially under the action of strong excitonic effects [14-19]. These effects are also very strong in ultrathin $(\lesssim 2 \mathrm{~nm})$ layers of semiconductors synthesized by colloidal chemistry, often called nanoplatelets [20,21].

These observations have inspired theoretical attempts to identify the origin of the $\pi \alpha$ quantization and its intrinsic limits [22-28]. In the case of graphene, deviations from $\pi \alpha$ appear when electrons lose their Dirac fermion character as a non-zero mass, for example at high energy relative to the Dirac point [26-28]. The same effect appears when a gap is opened at the Dirac point, for example under the action of spin-orbit coupling (SOC), as predicted in the case of silicene, germanene and stanene [25], and as shown in Sect. S8 of the Supplemental Material [29] (see, also, Refs. [30-33] therein).

All previous theoretical studies [22-28] were based on two-band models from which it was concluded that the $\pi \alpha$ quantization of the absorptance results from a numerical compensation between the joint density-of-states (JDOS) and the oscillator strength. However, this cancellation of terms is only perfect in the case of pristine graphene, for which the Fermi velocity determines both the band dispersion and the momentum matrix elements over a large energy range [5-10, 28]. In the case of quantum wells of zinc-blende semiconductors which are char- 
TABLE I. Summary of the results for the different types of materials considered in this work. CB and VB stand for conduction and valence bands, respectively. The number of bands per valley must be multiplied by two if spin is taken into account. In the case of Ge, we consider the direct bands at $\Gamma$. The integer $n>0$ indicates that the absorptance present several plateaus, multiple of $\pi \alpha$ or $2 \pi \alpha$.

\begin{tabular}{|c|c|c|c|c|c|c|c|}
\hline Material & Lattice & Valleys & $\begin{array}{c}\text { Number of bands } \\
\text { per valley }\end{array}$ & Band dispersion & $\mathrm{SOC}$ & $\begin{array}{c}\text { Absorptance } \\
\text { plateaus } \\
\end{array}$ & $\begin{array}{c}\text { Unit } \\
\text { per valley }\end{array}$ \\
\hline Graphene & Honeycomb & $\mathrm{K}(\times 2)$ & CB:1 VB:1 & Linear & No & $\pi \alpha$ & $\pi \alpha / 2$ \\
\hline $\begin{array}{l}\text { III-V or II-VI } \\
\text { semiconductor } \\
\text { Ge }\end{array}$ & $\begin{array}{l}\text { Zinc-blende } \\
\text { Diamond }\end{array}$ & $\begin{array}{l}\Gamma(\times 1) \\
\Gamma(\times 1)\end{array}$ & $\mathrm{CB}: 1 \mathrm{VB}: 3$ & $\begin{array}{l}\text { CB: Parabolic at the edge, } \\
\text { non-parabolic beyond } \\
\text { VB: Complex, } \\
\text { anisotropy, warping }\end{array}$ & $\begin{array}{l}\text { Yes, sets VB } \\
\text { SOC splitting }\end{array}$ & $\sim n \pi \alpha$ & $\sim \pi \alpha$ \\
\hline $\begin{array}{c}\text { IV-VI } \\
\text { semiconductor }\end{array}$ & Rocksalt & $\mathrm{L}(\times 4)$ & $\mathrm{CB}: 1 \mathrm{VB}: 1$ & $\begin{array}{l}\text { Parabolic at edges, } \\
\text { almost linear beyond }\end{array}$ & $\begin{array}{c}\text { Yes, } \\
\text { opens the gap }\end{array}$ & $\sim 2 n \pi \alpha$ & $\sim \pi \alpha / 2$ \\
\hline
\end{tabular}

acterized by heavy, light and split-off hole bands with marked anisotropies that couple under the effect of the confinement, a two-band model is not justified [34]. Previous theoretical studies $[13,23,24,26]$ assumed a constant JDOS, typical of two-dimensional (2D) materials with parabolic bands. In reality, however, InAs is characterized by marked non-parabolic effects due to a very strong coupling between valence and conduction bands [35-37]. As a result, the fundamental physical reasons for the $\pi \alpha$ quantization of the absorptance in InAs quantum wells remains largely to be revealed, and so does the universality of this characteristics. In this paper, we show that absorptance quantization can be found in 2D materials characterized by very different band structures, such as graphene, group-IV, III-V and II-VI semiconductors, and even in IV-VI semiconductors with a rocksalt lattice.

In the following, we present detailed atomistic tightbinding calculations of electronic structures and absorptance spectra of semiconductor quantum wells. As summarized in Table I, we predict that, despite band structures of great variety and complexity, quantum wells of many semiconductors are characterized by simple absorptance spectra with marked plateaus, directly interpretable in terms of the universal constant $\pi \alpha$. We show that InAs is not an singular example, but is on the contrary representative of other conventional semiconductors with weak excitonic effects, which is already the case for InAs layers thicker than $3 \mathrm{~nm}$ [13]. These predictions are reinforced by published absorption measurements in semiconductor superlattices whose results we revisit [23].

We show that this universality of the absorptance quantization has a common fundamental origin that we are able to reveal through simple analytical models. The nature of the electronic states involved in the optical transitions plays essentially only on the prefactor in front of $\pi \alpha$, which differs according to the categories of materials. This universal behavior thus provides simple rules for the design of photodetectors based on semiconductor quantum wells. It creates an unexpected bridge with the world of graphene, not by coincidence but due to very fundamental reasons. Finally, we remark that reports show that the fine structure constant also rules spontaneous emission and gain, a topic beyond the scope of the present work [23].

\section{METHODOLOGY}

\section{A. Expression for the absorptance}

The absorptance $\mathcal{A}$ is the measure of the absorbed light flux, such that $\mathcal{R}+\mathcal{A}+\mathcal{T}=1$ where $\mathcal{T}$ is the transmittance and $\mathcal{R}$ is the reflectance. In the case of a single quantum well deposited on a transparent substrate, the absorptance at photon energy $\hbar \omega$ can be written as

$$
\mathcal{A}(\hbar \omega)=F^{2} \mathcal{A}_{0}(\hbar \omega)
$$

where $F$ is equal to $2 /\left(n_{s}+1\right)$ in which $n_{s}$ is the refractive index of the substrate (assumed to be very thick). The prefactor $F^{2}$, which is a local-field factor, describes the fact that the quantum well experiences interfering incident and reflected electric fields. In the case of a sample irradiated perpendicularly, a quantum well being a quasi$2 \mathrm{D}$ system, the external electric field (plus its reflected component) is unscreened (for wavelength $\gg$ thickness), the polarization charges being repelled at the edges.

The rate for an optical transition between a valence subband $v$ and a conduction subband $c$ at wavevector $\mathbf{k}$ is determined by the oscillator strength $f_{c, v}(\mathbf{k})=$ $\left(2 / m_{0}\right)|\langle c, \mathbf{k}|\mathbf{p} \cdot \mathbf{e}| v, \mathbf{k}\rangle|^{2} /\left[E_{c}(\mathbf{k})-E_{v}(\mathbf{k})\right]$ where $\mathbf{e}$ is the light polarization vector, $\mathbf{p}$ is the momentum operator, $m_{0}$ is the free electron mass, $E_{c}(\mathbf{k})\left[E_{v}(\mathbf{k})\right]$ is the energy in the conduction [valence] subband. In absence of excitonic effects, the bare absorptance of a quantum well is given by $[38,39]$

$$
\mathcal{A}_{0}(\hbar \omega)=\frac{\pi \hbar e^{2}}{2 m_{0} c \varepsilon_{0} S} \sum_{c, v, \mathbf{k}} f_{c, v}(\mathbf{k}) \delta\left(\hbar \omega-E_{c}(\mathbf{k})+E_{v}(\mathbf{k})\right)
$$


where $S$ is the area of the sample on which we apply periodic boundary conditions that define the components of $\mathbf{k}$. For numerical implementation, the number of $\mathbf{k}$ vectors is increased until the results are converged, the Dirac delta function $\delta(x)$ in Eq. (2) being replaced by a Gaussian function $\exp \left[-x^{2} /\left(2 \eta^{2}\right)\right] /(\eta \sqrt{2 \pi})$ where $\eta$ characterizes all sources of broadening. Unless otherwise specified, $\eta$ is fixed at $10 \mathrm{meV}$.

\section{B. Tight-binding calculations of the absorptance}

The electronic structure of (001) quantum wells is calculated using an atomistic tight-binding method. Each atom of the compound is described by a set of 20 atomic orbitals, $s p^{3} d^{5} s^{*}$ for each spin degree of freedom where $s^{*}$ represents a second $s$ orbital. The dangling bonds at quantum well surfaces are saturated with pseudo-hydrogen atoms described by a single $s$ orbital [38], except for PbSe for which it is not necessary [40]. The Hamiltonian matrix elements are restricted to the nearest-neighbor interactions and onsite terms. Spinorbit coupling is included. We used the tight-binding model of Ref. 41 for InAs, Ref. 40 for PbSe, Ref. 42 for (zinc-blende) CdSe, Ref. 43 for GaAs, Ref. 44 for Ge, Ref. 45 for $\mathrm{HgTe}$. We have verified that very similar results have been obtained with other sets of parameters for PbSe [46] and for Ge [47].

Following a well-established recipe for the description in tight-binding of the optical properties of semiconductors, $[38,48]$ the momentum matrix elements are determined by those of the Hamiltonian $\mathbf{H}(\mathbf{k})$,

$$
\langle c, \mathbf{k}|\mathbf{p} \cdot \mathbf{e}| v, \mathbf{k}\rangle \rightarrow \frac{m_{0}}{\hbar}\langle c, \mathbf{k}|\nabla H(\mathbf{k}) \cdot \mathbf{e}| v, \mathbf{k}\rangle
$$

in which the gradient of $\mathbf{H}(\mathbf{k})$ is relative to $\mathbf{k}$.

\section{Condition for the existence of plateaus in the absorptance}

It is interesting to consider the case where the system is isotropic, i.e., where all quantities depend only on $k$, the modulus of $\mathbf{k}$. As shown in Appendix A, and as already found by Huang et al. [28], the bare absorptance can be written from Eq. (A4) as

$$
\mathcal{A}_{0}(\hbar \omega)=2 \pi \alpha \sum_{c, v} \frac{|\langle c, \mathbf{k}|\nabla H(\mathbf{k})| v, \mathbf{k}\rangle|^{2}}{\partial\left|E_{v}(k)-E_{c}(k)\right|^{2} / \partial\left(k^{2}\right)}
$$

with $k$ such that $E_{c}(k)-E_{v}(k)=\hbar \omega . \nabla H$, the gradient of the Hamiltonian with respect to $k$, comes from the expression of the momentum [Eq. (3)]. The numerator and denominator of Eq. (4) have thus the same unit, i.e., (energy.length $)^{2}$. Within one constant, the inverse of the

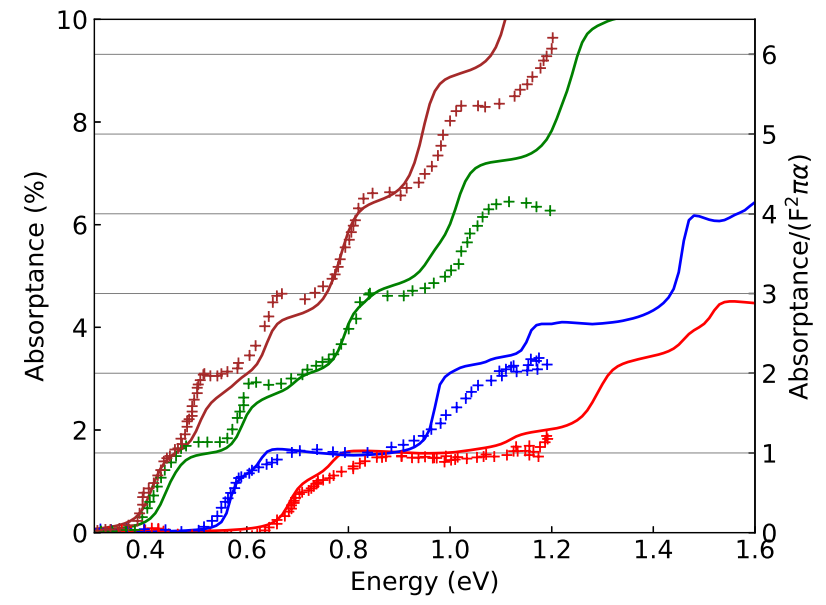

FIG. 1. Crosses: Measured absorptance [13] for InAs quantum wells of different thickness, $6.0 \mathrm{~nm}$ (red), $9.0 \mathrm{~nm}$ (blue), $14 \mathrm{~nm}$ (green), $19 \mathrm{~nm}$ (brown). Solid lines: absorptance $\mathcal{A}(\hbar \omega)$ calculated for quantum wells with slightly smaller thickness, $4.0 \mathrm{~nm}$ (red), $6.0 \mathrm{~nm}$ (blue), $12.5 \mathrm{~nm}$ (green), $17.5 \mathrm{~nm}$ (brown). Broadening: $\eta=20 \mathrm{meV}$.

numerator is equal to the joint density of states divided by $\hbar \omega$ (Appendix A).

Equation (4) shows that, for the absorptance to be constant, there must be compensation between the optical matrix element $|\langle c, \mathbf{k}|\nabla H(\mathbf{k})| v, \mathbf{k}\rangle|^{2}$ and the denominator. As we show in the following, this is likely to occur when a non-diagonal term of the Hamiltonian, linear in $k$ ( $\sim \mathbf{V} \cdot \mathbf{k}$ where $\mathbf{V}$ is a constant vector $)$, coupling conduction and valence subbands, dominates. In this case, both the numerator and denominator are proportional to $|\mathbf{V}|^{2}$ and $\mathcal{A}_{0}(\hbar \omega)$ is a constant determined by the symmetry of the electronic states.

\section{RESULTS AND DISCUSSION}

\section{A. InAs}

Figure 1 presents the absorptance for InAs quantum wells. The results of the calculations are compared with the experimental data of Ref. 13. We have adjusted the thickness of the quantum wells in order to obtain the correct optical threshold (gap) compared to experiments. A reduction in thickness of the order of $2 \mathrm{~nm}$ is needed (Fig. 1), which can be understood by band-bending effects which are well-known in the case of InAs surfaces, due to the pinning of the Fermi level by surface states [49].

The agreement between theory and experiments in Fig. 1 is quite remarkable, showing that excitonic effects which are not taken into account in our calculations are weak in these InAs quantum wells. The absorptance is characterized by very clear steps. Their position, their amplitude, and even their shape when they exhibit fine 

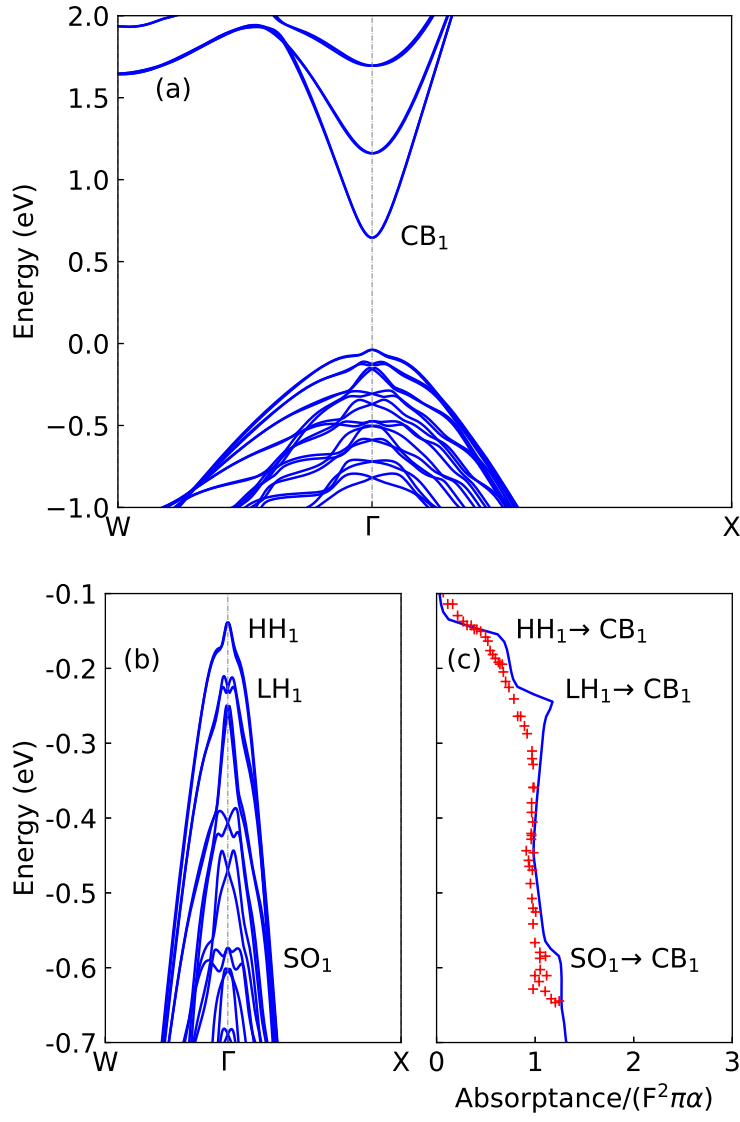

FIG. 2. (a) Lowest conduction subbands and highest valence subbands of a $4 \mathrm{~nm}$ thick InAs quantum well. (b) Zoom on the highest valence subbands and, facing each other, (c) plot of the bare absorptance calculated with a smaller broadening $(\eta=6 \mathrm{meV})$ than in Fig. 1 in such a way that each absorptance plateau can be associated to a transition from a valence subband $\left(\mathrm{HH}_{1}, \mathrm{LH}_{1}, \mathrm{SO}_{1}\right)$ to the lowest conduction subband $\left(\mathrm{CB}_{1}\right)$. Red crosses: experiments [13] for a $6 \mathrm{~nm}$ thick quantum well. High symmetry points of the Brillouin zone: $\mathrm{W}=\pi / a[1,1,0], \mathrm{X}=2 \pi / a[1,0,0], a$ being the bulk lattice parameter.

structures are well described by the calculations. The main plateaus in the spectra correspond to a $\pi \alpha$ quantization of the bare absorptance $\mathcal{A}_{0}(\hbar \omega)$.

As shown in Fig. 2a for a $4 \mathrm{~nm}$ quantum well, the band structure is composed of subbands due to the vertical quantum confinement effect. The valence subbands (Fig. 2b) exhibit a complex dispersion, due to the contribution of the Heavy-Hole (HH), Light-Hole ( $\mathrm{LH})$ and Split-Off (SO) bands [34]. In spite of this complexity, the absorptance shown in Fig. 1 has relatively simple behavior, with main plateaus of $\mathcal{A}_{0}(\hbar \omega)$ at $n \pi \alpha$ with $n \in \mathcal{N}$ (for additional results for a thickness of $12.5 \mathrm{~nm}$, see Fig. S1 of Supplemental Material [29]).

Figure 2c presents the absorptance calculated using a smaller broadening to reveal the details. This shows that the first plateau is in fact composed of three steps

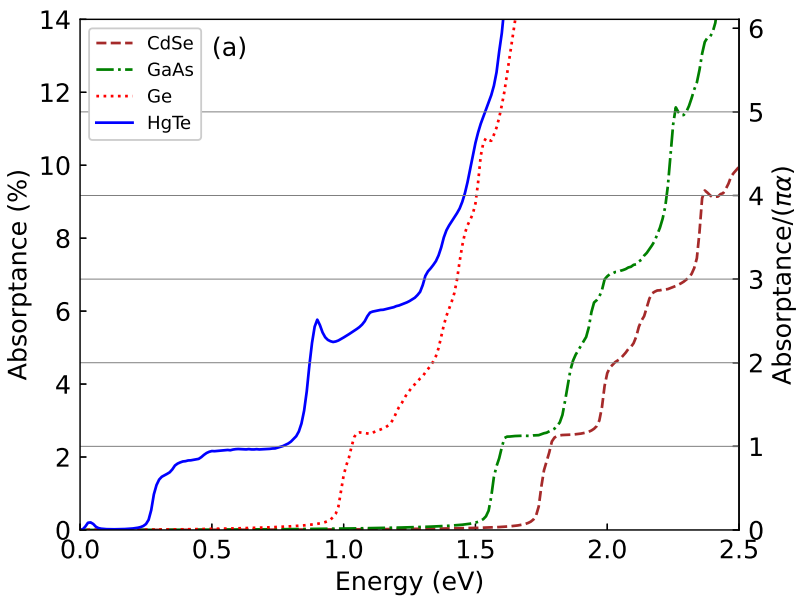

FIG. 3. Bare absorptance $\mathcal{A}_{0}(\hbar \omega)$ calculated for $6 \mathrm{~nm}$ thick quantum wells of CdSe (brown dashed line), Ge (red dotted line), GaAs (green dotted-dashed line), and for a $3.9 \mathrm{~nm}$ thick quantum well of $\mathrm{HgTe}$ (blue solid line).

corresponding to the transitions from valence subbands $\mathrm{HH}_{1}, \mathrm{LH}_{1}$ and $\mathrm{SO}_{1}$ to the lowest conduction subband $\mathrm{CB}_{1}$ (Fig. 2a and Fig. 2b). Here the index 1 represents the first quantum mode induced by the vertical confinement in the quantum well. Similarly, a detailed analysis shows that the plateau at $n \pi \alpha$ can be associated with transitions $(\mathrm{HH}+\mathrm{LH}+\mathrm{SO})_{n} \rightarrow \mathrm{CB}_{n}$ (Supplemental Material [29], Fig. S1 in Sect. S1).

It is important to point out that the description of the valence subbands in the form $\mathrm{HH}_{n}, \mathrm{LH}_{n}$ and $\mathrm{SO}_{n}$ is only a simplified representation. Indeed, it is not easy to follow the $\mathrm{HH}, \mathrm{LH}, \mathrm{SO}$ character of the subbands as a function of $k$ because of their non-parabolicity, their warping, and their $k$-dependent mixing under quantum confinement, which lead to complex dispersion including band crossings and anti-crossings (Fig. 2b). However, as shown in Appendix B and in the following sections, this simple representation is justified by extracting a $2 \mathrm{D} \mathbf{k} \cdot \mathbf{p}$ model from the atomistic tight-binding calculations. In other words, this very common description [34] of the bands in terms of $\mathrm{HH}, \mathrm{LH}$ and $\mathrm{SO}$ is well justified as long as we only want to describe the main optical transitions which tend to hide the subtle features of the band structure.

\section{B. Other semiconductors}

InAs is a semiconductor characterized by a small band gap. It is therefore interesting to test the quantization of absorption in the case of semiconductors with a higher band gap. Fig. 3 shows that the absorptance spectra of GaAs and CdSe quantum wells are also characterized by several well-marked steps close to multiples of $\pi \alpha$. The deviations from $\pi \alpha$ are here larger than for InAs but remain relatively modest. 


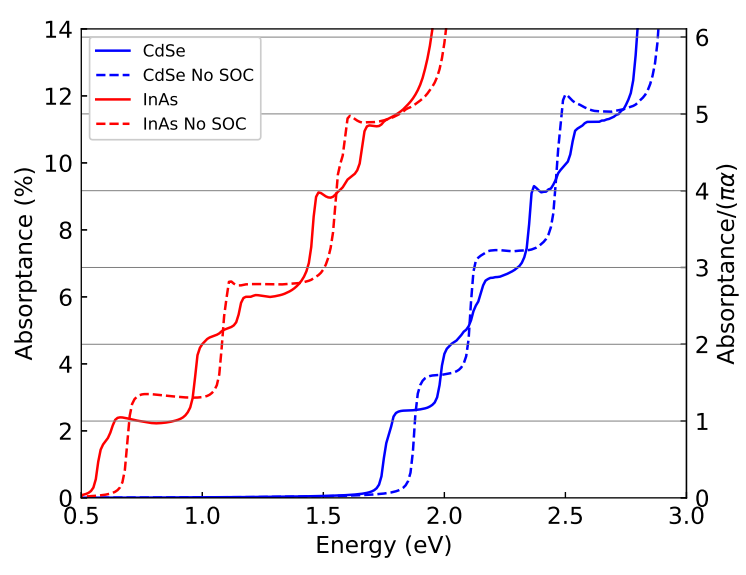

FIG. 4. Bare absorptance $\mathcal{A}_{0}(\hbar \omega)$ calculated in tight-binding for 6-nm-thick layers of InAs (red curves) or CdSe (blue curves), with (solid lines) or without (dashed lines) SOC.

Remarkably, the first step of $\mathcal{A}_{0}(\hbar \omega)$ at $\approx \pi \alpha$ is also clearly visible in the case of a Ge quantum well, yet an indirect semiconductor. In fact, in bulk Ge, the minimum of the conduction band at $\Gamma$ is just above the minima at $\mathrm{L}$ points, so the first step corresponds to the vertical transitions in the vicinity of $\Gamma$. At higher energy, the steps are less visible, due to the complex conduction band structure of Ge in this region [50].

Another particularly interesting system is $\mathrm{HgTe}$, a semi-metal, which can be seen as an inverted band semiconductor due to the strong SOC. Under the effect of a weak quantum confinement, a band gap opens, leading to the formation of a topological insulator as long as band remains inverted [51-53]. When the thickness of the layers is reduced, the band gap closes and then opens again. In this case, the band order of a conventional semiconductor is restored. Remarkably, a band gap of the order of one electron-volt can even be obtained in ultrathin layers, colloidal nano-platelets [54]. We have therefore considered a $3.9 \mathrm{~nm}$ thick (001) HgTe quantum well, characterized by a band gap of $0.19 \mathrm{eV}$, thin enough to be in the non-inverted regime, thick enough to minimize the excitonic effects. Figure 3 shows that the absorptance spectrum is again characterized by a very marked plateau at $\pi \alpha$. However, this plateau is preceded by several intermediate steps, which are relatively long, but whose contributions converge towards $\pi \alpha$. These steps, as well as the sharp peaks visible at higher energy, are due to the complex energy dispersion of the valence subbands with heavy-hole and light-hole components (Supplemental Material [29], Fig. S2).

\section{Role of spin-orbit coupling}

Figure 4 illustrates the effect of the SOC. In the absence of SOC, $\mathcal{A}_{0}(\hbar \omega)$ in InAs and CdSe quantum wells is still quantized, but the height of the first plateau is closer to $1.5 \pi \alpha$ than $\pi \alpha$. This is also true for the following steps which are clearly larger than $\pi \alpha$. When the $\mathrm{SOC}$ is restored, the quantization in $\pi \alpha$ becomes remarkable.

In the following, in order to understand these behaviors, we will work in two steps, first, by proposing an analytical model of $\mathcal{A}_{0}(\hbar \omega)$ in the absence of SOC, second, by understanding the effect of SOC on the absorptance step height.

\section{FUNDAMENTALS OF THE ABSORPTANCE PLATEAUS}

\section{A. The prototypical model of graphene}

To illustrate the compensation mechanism in Eq. (4), it is interesting to return to the case of graphene to understand what is happening in semiconductor quantum wells. In each valley $K$ or $K^{\prime}$ of graphene, the Hamiltonian matrix near the Dirac point (zero of energy) can be written, in the basis of the Bloch functions made of $p_{z}$ orbitals on the two sublattices of the honeycomb, as follows [55]

$$
H=\left[\begin{array}{cc}
0 & V\left(k_{x}+i k_{y}\right) \\
V^{*}\left(k_{x}-i k_{y}\right) & 0
\end{array}\right]
$$

where $V=\hbar v_{F}$ is related to the Fermi velocity $v_{F}$.

By defining bottom $\left(\Psi_{-}\right)$and top $\left(\Psi_{+}\right)$band states, the numerator and the denominator of Eq. (4) are given by

$$
\begin{aligned}
& \left|\left\langle\Psi_{+}|\nabla H(\mathbf{k})| \Psi_{-}\right\rangle\right|^{2}=|V|^{2} \\
& \partial\left|E_{+}(k)-E_{-}(k)\right|^{2} / \partial k^{2}=4|V|^{2}
\end{aligned}
$$

There is a total compensation between these two terms in Eq. (4), so that $|V|^{2}$ disappear [28]. The bare absorptance per valley is therefore $\mathcal{A}_{0}(\hbar \omega)=\pi \alpha / 2$, independent of $V$ and any material characteritics [5-10].

This compensation is no longer effective in the case of massive Dirac bands (Sect. S8 in Supplemental Material [29] and Refs. [25-28]).

\section{B. A prototypical analytical model for semiconductor quantum wells}

In this section, we present a three-band model based on the Kane Hamiltonian [56] that provides the simplest description of the absorptance in III-V or II-VI semiconductor quantum wells, in absence of SOC. $\mathcal{A}_{0}(\hbar \omega)$ is calculated analytically as the sum of two components, the first of which is equal to $\pi \alpha$, the second depends only on the quantum well gap energy and tends to zero at high 
energy. This model thus sheds light on the fundamental origins of the quantization of the absorptance and its relative invariance from one semiconductor to another.

The model Hamiltonian $H$ is defined in a basis of three vectors, corresponding to the Bloch states at $\mathbf{k}=0$, one of $s$ character $(|s\rangle)$ for the conduction band, two of $p$ character $(|x\rangle,|y\rangle)$ for valence bands, translating the $2 \mathrm{D}$ character of the system. Keeping only the principal coupling terms at the first-order in $k(\propto P)$, we write

$$
H=\left[\begin{array}{ccc}
E_{g} & i P k_{x} & i P k_{y} \\
-i P k_{x} & 0 & 0 \\
-i P k_{y} & 0 & 0
\end{array}\right]
$$

where $E_{g}$ is the energy gap of the quantum well. Introducing two vectors,

$$
\begin{aligned}
& \left|p_{\|}\right\rangle=\frac{k_{x}|x\rangle+k_{y}|y\rangle}{k}, \\
& \left|p_{\perp}\right\rangle=\frac{k_{y}|x\rangle-k_{x}|y\rangle}{k},
\end{aligned}
$$

the Hamiltonian in the basis $\left\{|s\rangle,\left|p_{\|}\right\rangle,\left|p_{\perp}\right\rangle\right\}$ becomes

$$
H=\left[\begin{array}{ccc}
E_{g} & i P k & 0 \\
-i P k & 0 & 0 \\
0 & 0 & 0
\end{array}\right]
$$

The solutions are $\left|\Psi_{h h}\right\rangle=\left|p_{\perp}\right\rangle$ of energy $E_{h h}=0$ (flat band), and the states $\left|\Psi_{e}\right\rangle$ and $\left|\Psi_{l h}\right\rangle$ of energy

$$
E_{l h}(k)=\left(\frac{E_{g}}{2}\right) \pm \sqrt{\left(\frac{E_{g}}{2}\right)^{2}+P^{2} k^{2}}
$$

respectively. $E_{e}(k)$ is the energy of the conduction subband. $E_{h h}(k)$ and $E_{l h}(k)$ are the energies of $\mathrm{HH}$ and $\mathrm{LH}$ subbands, respectively. Interestingly, the energy dispersions in this model are basically the same as for massive Dirac fermions.

The absorptance coming from transitions $\left|\Psi_{h h}\right\rangle \rightarrow$ $\left|\Psi_{e}\right\rangle$ and $\left|\Psi_{l h}\right\rangle \rightarrow\left|\Psi_{e}\right\rangle$ is written as $\mathcal{A}_{0}(\hbar \omega)=$ $\mathcal{A}_{h h-e}(\hbar \omega)+\mathcal{A}_{l h-e}(\hbar \omega)$ and is calculated analytically using Eq. (4), replacing $(|v, \mathbf{k}\rangle,|c, \mathbf{k}\rangle)$ by $\left(\left|\Psi_{h h}\right\rangle,\left|\Psi_{e}\right\rangle\right)$ or $\left(\left|\Psi_{l h}\right\rangle,\left|\Psi_{e}\right\rangle\right)$, respectively. Details on the calculations are given in Supplemental Material [29], Sect. S4.

\section{Calculation of the absorptance for the heavy-hole electron transition}

For the transition $\Psi_{h h} \rightarrow \Psi_{e}$ at the energy $\hbar \omega=$ $E_{e}(k)-E_{h h}(k)$, the optical matrix element is given by (Supplemental Material [29], Sect. S4.1)

$$
\left|\left\langle\Psi_{e}|\nabla H(\mathbf{k})| \Psi_{h h}\right\rangle\right|^{2}=\frac{P^{2} \hbar \omega}{2 \hbar \omega-E_{g}}
$$

and the denominator of Eq. (4) by

$$
\frac{\partial\left|E_{e}(k)-E_{h h}(k)\right|^{2}}{\partial k^{2}}=\frac{2 P^{2} \hbar \omega}{2 \hbar \omega-E_{g}} .
$$

We deduce the absorptance

$$
\mathcal{A}_{h h-e}(\hbar \omega)=\pi \alpha \theta\left(\hbar \omega-E_{g}\right)
$$

where $\theta(x)$ is the unit step function.

Remarkably, as in the case of graphene, there is a complete compensation between the different terms so that the absorptance is a constant, independent of $P, E_{g}$, and $\hbar \omega$. This compensation takes place while the JDOS is not constant (Supplemental Material [29], Sect. S4.1), unlike the ideal case of $2 \mathrm{D}$ parabolic valence and conduction bands.

\section{Calculation of the absorptance for the light-hole electron transition}

For the transition $\Psi_{l h} \rightarrow \Psi_{e}$ at the energy $\hbar \omega=$ $E_{e}(k)-E_{l h}(k)$, the optical matrix element is given by (Supplemental Material [29], Sect. S4.2)

$$
\left|\left\langle\Psi_{e}|\nabla H| \Psi_{l h}\right\rangle\right|^{2}=\left(\frac{E_{g} P}{\hbar \omega}\right)^{2}
$$

and the denominator of Eq. (4) by

$$
\partial\left|E_{e}(k)-E_{l h}(k)\right|^{2} / \partial k^{2}=4 P^{2}
$$

The absorptance is thus given by

$$
\mathcal{A}_{l h-e}(\hbar \omega)=\frac{\pi \alpha}{2}\left(\frac{E_{g}}{\hbar \omega}\right)^{2} \theta\left(\hbar \omega-E_{g}\right)
$$

Also remarkably, this contribution does not depend on $P$, is equal to $\pi \alpha / 2$ when $\hbar \omega=E_{g}$, and tends to 0 when $\hbar \omega \gg E_{g}$.

$N B$ : This is at variance with graphene where the transition between the two band gives a plateau at $\pi \alpha / 2$. Note that, as in the case of graphene, the JDOS is proportional to $\hbar \omega$ [Eq. (S32) in Supplemental Material [29]]. The difference is therefore due to different local 2D symmetries, i.e., to the different nature of the Bloch states. 


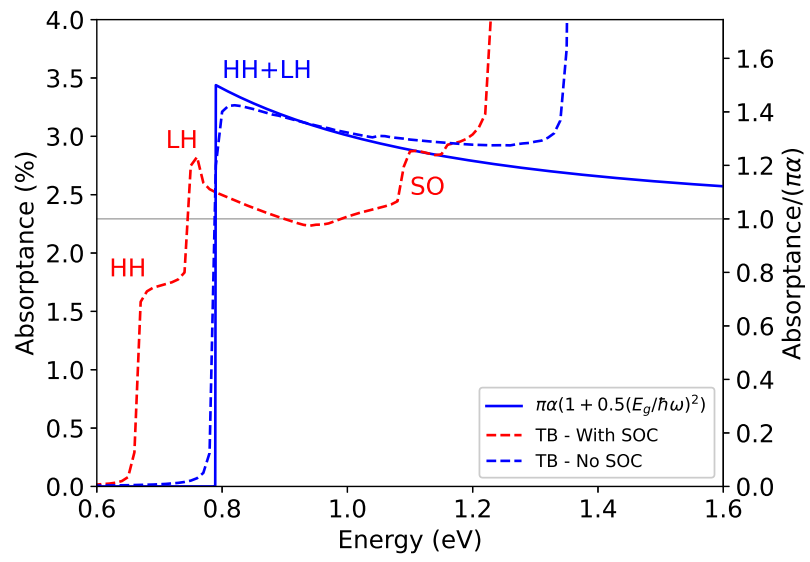

FIG. 5. Bare absorptance $\mathcal{A}_{0}(\hbar \omega)$ for 4.2 -nm-thick layer of InAs calculated in tight-binding (TB, dashed curves) with (red) or without (blue) SOC. The blue solid line shows $\mathcal{A}_{0}(\hbar \omega)$ given by the simple analytical model, Eq. (18) with $E_{g}=$ $0.79 \mathrm{eV}$. For the sake of comparison, the tight-binding calculations were performed with a small broadening $\eta$ of $2 \mathrm{meV}$.

\section{Comparison to tight-binding calculations}

The total absorptance in this simple model is therefore given by

$$
\mathcal{A}_{0}(\hbar \omega)=\pi \alpha\left[1+\frac{1}{2}\left(\frac{E_{g}}{\hbar \omega}\right)^{2}\right] \theta\left(\hbar \omega-E_{g}\right) .
$$

Figure 5 shows that, despite its great simplicity, the analytical model reproduces remarkably well the results obtained by the tight-binding method, in the absence of SOC. This model describes the essential characteristics of the optical transitions from valence subbands to the first conduction subband. It gives a simple explanation to the quantization of the absorptance, and to its universal character since the Eq. (18) only involves the energy gap as a characteristic quantity of the semiconductor quantum well. As in the case of graphene, $\pi \alpha$ is a metric of the absorptance.

\section{Justification of the simple analytical model}

We have shown in the previous sections that the valence band structure of III-V or II-VI semiconductor quantum wells is in general of great complexity. In this context, it is not obvious that a three-band model, including one flat band and two fully symmetric bands is representative of real systems.

The general justification of a $2 \mathrm{D} \mathbf{k} \cdot \mathbf{p}$ model, starting from a bulk Kane Hamiltonian, is presented in Supplemental Material [29], Sect. S3. We also present and justify in Appendix B a more elaborate $(3 \times 3) 2 \mathrm{D} \mathrm{k} \cdot \mathbf{p}$ model

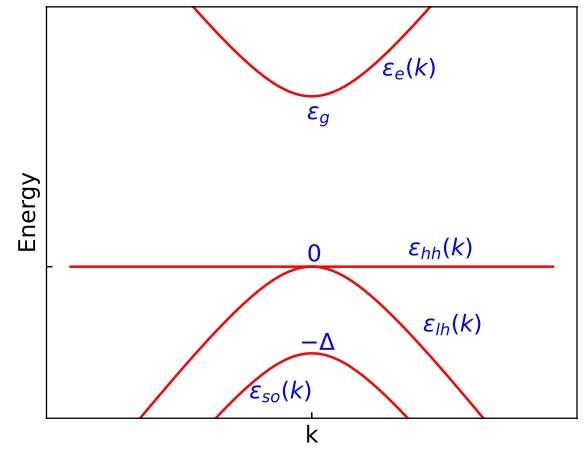

FIG. 6. Band structure in the analytical model including SOC. The energies are noted by the Greek letter $\varepsilon$ instead of $E$ to distinguish from the case without SOC.

whose parameters are directly deduced from tight binding calculations, following the methodology described in Supplemental Material [29], Sect. S5. This model includes all matrix elements up to the second order in components of $\mathbf{k}$. We deduce from Appendix B that the simple analytical model described above is justified because the terms of the form $P k_{x}$ and $P k_{y}$ of the $2 \mathrm{D} \mathbf{k} \cdot \mathbf{p}$ Hamiltonian dominate in the expression of the different components of the absorptance. The simple analytical model is justified even more when the energy gap of the semiconductor is small. The deviations from the absorption values predicted by Eq. (18) remain nevertheless small even for large gap materials.

\section{Influence of the SOC on the absorptance}

We have seen in Fig. 4 and Fig. 5 that the SOC contributes to bring the plateaus from about $1.5 \pi \alpha$ (at $E_{g}$ ) to about $\pi \alpha$. Here we discuss the reasons, on the one hand with the help of a simplest-as-possible analytical model describing the main underlying effects, on the other hand by analyzing the results of the tight binding calculations

\section{A prototypical analytical model including $S O C$}

We start from the Kane Hamiltonian [56] including SOC in the basis of Bloch states $|s \uparrow\rangle,|x \uparrow\rangle,|y \uparrow\rangle$, $|z \uparrow\rangle,|s \downarrow\rangle,|x \downarrow\rangle,|y \downarrow\rangle,|z \downarrow\rangle$, where $O z$ remains perpendicular to the quantum well. We write the Hamiltonian as two identical $4 \times 4$ blocks [34]

$$
H=\left[\begin{array}{cccc}
\varepsilon_{g} & i \sqrt{\frac{2}{3}} P k & -\frac{i}{\sqrt{3}} P k & 0 \\
-i \sqrt{\frac{2}{3}} P k & 0 & 0 & 0 \\
\frac{i}{\sqrt{3}} P k & 0 & -\Delta & 0 \\
0 & 0 & 0 & 0
\end{array}\right]
$$


for one block in the basis $|s \uparrow\rangle,\left|\frac{3}{2}, \frac{1}{2}\right\rangle,\left|\frac{1}{2}, \frac{1}{2}\right\rangle,\left|\frac{3}{2}, \frac{3}{2}\right\rangle$, for the other one in the basis $|s \downarrow\rangle,\left|\frac{3}{2},-\frac{1}{2}\right\rangle,\left|\frac{1}{2},-\frac{1}{2}\right\rangle$, $\left|\frac{3}{2},-\frac{3}{2}\right\rangle$, eigenvectors of $\mathbf{J}$ and $J_{Z}$ with $Z$ oriented along k. $\varepsilon_{g}$ is the gap in presence of SOC, and $\Delta$ is the splitting between $J=3 / 2$ and $J=1 / 2$ states due to the SOC. The expression of the $J=3 / 2$ and $J=1 / 2$ states in terms of $|x \uparrow\rangle \ldots|z \downarrow\rangle$ is given in Sect. S7 of the Supplemental Material [29].

In the same spirit as before, following Sect. S3 of the Supplemental Material [29], we treat the 2D problem with $k_{z}=0\left(k \equiv k_{\|}\right)$. The corresponding band structure is shown schematically in Fig. 6. The heavy-hole band is flat $\left[\varepsilon_{h h}(k)=0\right]$ and is degenerate at $k=0$ with the light-hole band $\left[\varepsilon_{l h}(k)\right]$. The electron band $\varepsilon_{e}(k)$ starts at $\varepsilon_{g}$ at $k=0$, the top of the split-off band $\varepsilon_{s o}(k)$ is at $-\Delta$.

Therefore in this model, the absorptance spectrum exhibits two steps, the first one at $\hbar \omega=\varepsilon_{g}$ that include two contributions (heavy and light holes $\rightarrow$ electrons), the second one at $\hbar \omega=\varepsilon_{g}+\Delta$ (split-off $\rightarrow$ electrons). As described in Sect. S7 of the Supplemental Material [29], the height of these steps can be calculated analytically at $k_{\|}=0$, the energy dispersions of the different bands being obtained by treating $P k$ terms within second order perturbation theory (becomes unvalid when $\Delta \rightarrow 0)$. The height of the first and second steps is given by

$$
\begin{aligned}
& \mathcal{A}_{0,1}\left(\varepsilon_{g}\right)=\pi \alpha\left[\frac{3}{4\left(1+\frac{\varepsilon_{g}}{2\left(\varepsilon_{g}+\Delta\right)}\right)}+\frac{1}{2\left(1+\frac{\varepsilon_{g}}{4\left(\varepsilon_{g}+\Delta\right)}\right)}(\$ 0)\right. \\
& \delta \mathcal{A}_{0,2}\left(\varepsilon_{g}+\Delta\right)=\pi \alpha\left[\frac{1}{2\left(1+\frac{\varepsilon_{g}+\Delta}{\varepsilon_{g}}\right)}\right]
\end{aligned}
$$

where $\delta \mathcal{A}_{0,2}$ in the second equation means that it must be added to the other contributions at $\hbar \omega=\varepsilon_{g}+\Delta$ to get the total absorptance.

Using the data corresponding to the InAs quantum well of $4.2 \mathrm{~nm}$ (Fig. 5), i.e., $\varepsilon_{g} \approx 0.7 \mathrm{eV}$ and $\Delta \approx 0.4 \mathrm{eV}$, the height of the first step [Eq. (20)] is about $\pi \alpha$, and the height of the second step [Eq. (21)] is about $0.2 \pi \alpha$, in excellent agreement with the tight binding results of Fig. 5 and the experimental data of Ref. 13. Remarkably, Eq. (20) and Eq. (21) only depend on $x=\varepsilon_{g} / \Delta$, and $\mathcal{A}_{0}\left(\varepsilon_{g}\right)$ is found to be very close to $\pi \alpha$ in a wide range of values of $x$. Figure S5 of the Supplemental Material [29] shows a deviation of Eq. (20) from $\pi \alpha$ below $10 \%$ for $x$ between 0.5 and 5 . This explains the universality of the $\pi \alpha$ quantization of the absorptance in presence of SOC.

\section{Comparison to tight-binding calculations}

Figure 5 allows to understand the effect of the SOC on the absorptance spectrum calculated in tight-binding. The first step found in absence of SOC transforms in
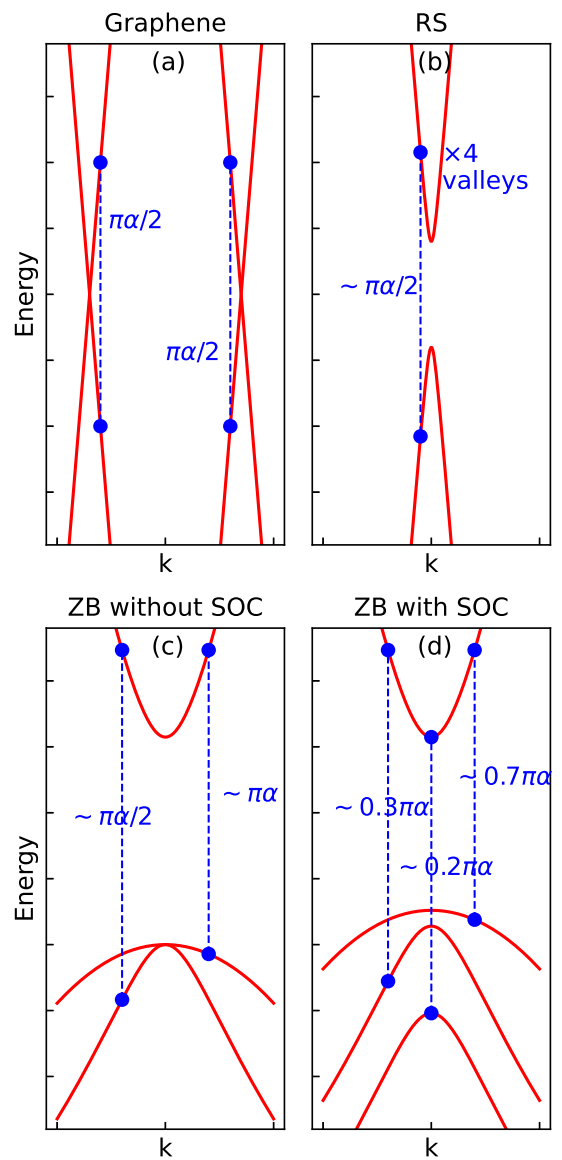

FIG. 7. Schematic representation of electronic bands and optical transitions in graphene (a), in a rocksalt (RS) semiconductor quantum well (b), in a zinc-blende (ZB) semiconductor quantum well without (c) or with (d) SOC. The intensity at the optical thresholds of the absorptance steps is indicated (the values are indicative, they may slightly vary from one material to another). The corresponding data are also summarized in Table I.

three steps. A short plateau at about $0.7 \pi \alpha$ is followed, after a small bump, by a long plateau close to $\pi \alpha$. This corresponds to transitions from $\mathrm{HH}$ and $\mathrm{LH}$ bands, respectively, which were degenerate at $k_{\|}=0$ by construction in the analytical model. Then, at higher energy $(\sim 1.1 \mathrm{eV})$, there is a third small step (split-off) such that the absorptance finally reaches the value obtained without SOC. Apart from the fact that it does not give a splitting between LH and HH bands, the analytical model accounts for this behavior very well.

Note that the respective weights of the steps from $\mathrm{HH}$ and LH depend significantly on the nature of the semiconductor, the spin-orbit coupling and the vertical confinement. 


\section{Summary of our understanding}

Figure 7 summarizes our understanding of the origin of absorptance quantization in III-V or II-VI semiconductor quantum wells and in graphene. This quantization comes from the compensation between numerator and denominator in Eq. (4). In the case of graphene, this compensation is exact which leads to an absorptance $\pi \alpha / 2$ for each of the two $K$ valleys.

In the case of a semiconductor quantum well, the situation seems at first sight very different because there are optical transitions between valence bands mainly derived from $p$ orbitals and a conduction band of strong $s$ character. However, under strong vertical confinement, in absence of SOC, the valence band can be described by two bands coupled to the conduction band by terms like $P k_{x}$ and $P k_{y}$. Transitions between the LH subband and the conduction subband lead to an absorptance of the order of $\pi \alpha / 2$ at the threshold, decreasing to zero at higher energy. Transitions between the $\mathrm{HH}$ subband and the conduction subband give a constant absorptance at $\pi \alpha$. In absence of SOC, the total absorptance is thus characterized by a single step at $\sim 3 \pi \alpha / 2$ which decreases to $\pi \alpha$ at higher energy (Fig. 5). This reasoning remains correct as long as $P^{2} / E_{g}$ is large compared to the other terms of the $\mathbf{k} \cdot \mathbf{p}$ Hamiltonian (Appendix B), which is the situation in many conventional semiconductors [34]. The height of the different contributions of the absorptance can vary from one compound to another, but the previous description remains nevertheless qualitatively correct for all the studied zinc-blende materials, especially those with a small bandgap.

When the SOC is switched on, the absorptance remains quantized because of the compensation effect due to the dominance of the coupling terms $P k_{i}$. However, the bands are split and a certain part of the oscillator strength is transferred to the SO band which is lower in energy. This leads to an absorptance $\approx 0.7 \pi \alpha$ for the transitions from $\mathrm{HH}$ subband, $\approx 0.3 \pi \alpha$ for those from LH subband, and $\approx 0.2 \pi \alpha$ for those from SO subband [Fig. $7(\mathrm{~d})]$. The total contribution from transitions from $\mathrm{HH}$ and LH subbands is thus close to $\pi \alpha$, which explains the wide plateau in Fig. 5 . Of course, the $\pi \alpha$ quantization of the absorptance is not exact, numbers slightly depend on the nature of the semiconductor, the plateaus are not totally flat because the compensation is not perfect, and therefore do not form a standard for the fine structure constant [24]. However, our calculations show that the quantization is universal, provided that excitonic effects can be neglected.

This quantification of the absorptance is visible as long as the energy gap between the $\pi \alpha$ steps remains smaller than the broadening factors. This gap becomes smaller and smaller as the thickness of the quantum well decreases. In III-V and II-VI materials, this gap is essentially determined by the quantum confinement in the conduction band and thus varies approximately as the inverse of the well thickness square.

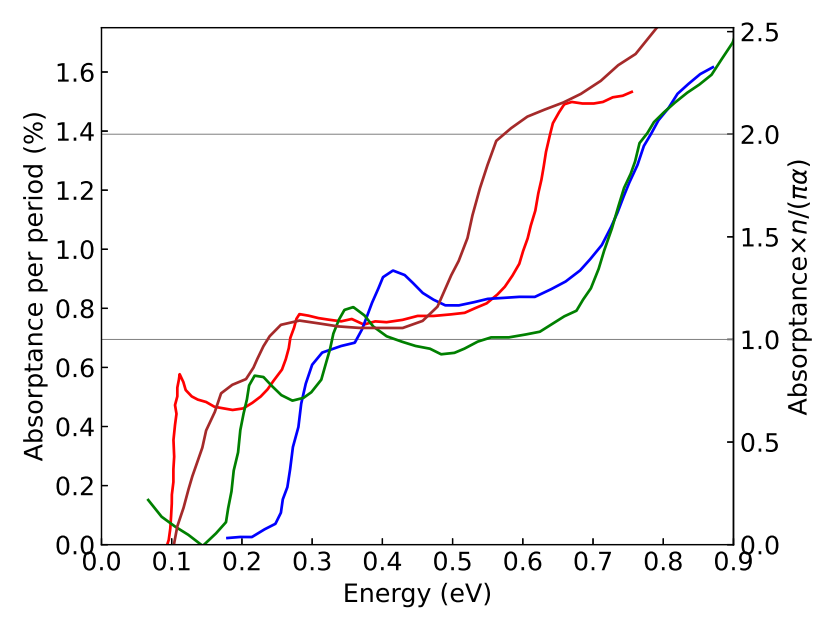

FIG. 8. Absorptance per period in $\mathrm{HgTe} / \mathrm{Hg}_{x} \mathrm{Cd}_{1-x} \mathrm{Te}$ superlattices deduced from the measurement of their absorption coefficient (period $=$ well thickness $L_{w}$ plus barrier thickness $L_{b}$ ). The vertical axis on the right gives the absorptance multiplied by the sample refractive index $n$ normalized by $\pi \alpha$. Red line: data of Ref. 57 for $x=0.05, L_{w}=4.15 \mathrm{~nm}$, $L_{b}=8.95 \mathrm{~nm}$. Brown line: data of Ref. 58 for $x=0.15$, $L_{w}=5.8 \mathrm{~nm}, L_{b}=4.2 \mathrm{~nm}$. Green line: data of Ref. 59 for $x=0.37, L_{w}=3.47 \mathrm{~nm}, L_{b}=7.60 \mathrm{~nm}$. Blue line: data of Ref. 60 for $x=0.32, L_{w}=3.4 \mathrm{~nm}, L_{b}=7.70 \mathrm{~nm}$.

\section{REVISITING EXPERIMENTAL DATA ON SUPERLATTICES}

The physical effects explaining the quantization of absorption should be operative also in semiconductor superlattices, provided that the barriers are high and the couplings between quantum wells are weak (multilayers). As already proposed in Ref. 23, this invites us to revisit experimental results obtained on these systems. Typically, the experiments consist of measuring the transmission through relatively thick layers composed of a large number (25-50) of periods, each formed by a quantum well and a barrier. The absorption coefficient $a(\hbar \omega)$ of the medium, deduced from these measurements, is given quite generally by

$$
a(\hbar \omega)=\frac{\omega}{c n} \varepsilon^{\prime \prime}(\hbar \omega)
$$

where $\varepsilon^{\prime \prime}$ is the imaginary part of the dielectric constant and $n$ is the refractive index of the medium. Consequently, the absorptance of a single period can be defined as

$$
\mathcal{A}(\hbar \omega)=a(\hbar \omega) L_{p}
$$

where $L_{p}=L_{w}+L_{b}$ is the length of the period, $L_{w}\left(L_{b}\right)$ being the thickness of the quantum well (barrier). However, we can deduce from Eq. (22) and the theory that we discussed above, that the quantity of interest, the one 


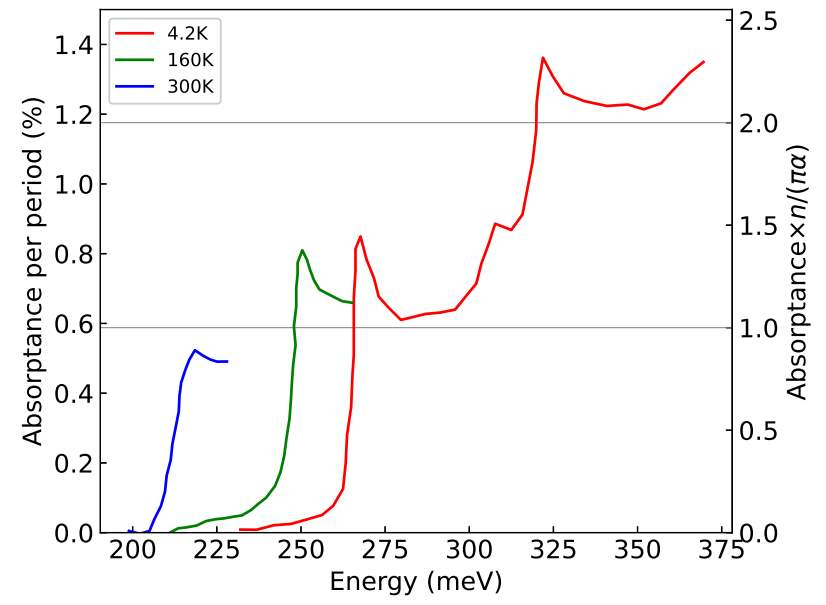

FIG. 9. Absorptance per period in $\mathrm{InSb} / \mathrm{Al}_{0.09} \mathrm{In}_{0.91} \mathrm{Sb}$ superlattices deduced from the measurement [61] of their absorption coefficient (well thickness $=22.5 \mathrm{~nm}$, barrier thickness $=$ $50 \mathrm{~nm}$ ) at different temperatures, $4.2 \mathrm{~K}$ (red line), 160K (green line), or $300 \mathrm{~K}$ (blue line). The right vertical axis gives the absorptance multiplied by the sample refractive index $(n=3.9)$ normalized by $\pi \alpha$. Spectra at $160 \mathrm{~K}$ and $300 \mathrm{~K}$ were measured in a limited energy range [61].

to be quantized in units of $\pi \alpha$, should be $n \mathcal{A}(\hbar \omega)$ [23]. In the following, we revisit experimental results from the literature, deliberately presenting them by increasing width of their band gap.

Figure 8 presents the absorptance per period which was measured in $\mathrm{HgTe} / \mathrm{Hg}_{x} \mathrm{Cd}_{1-x} \mathrm{Te}$ superlattices. The spectra are characterized by a wide plateau for which $n \mathcal{A}(\hbar \omega)$ is approximately equal to $\pi \alpha$. Just at the optical threshold, there is also a smaller step at approximately $3 \pi \alpha / 4$, which can be attributed to transitions to the heavy-hole subband. Interestingly, the absorptance spectrum that we predicted for a 3.9-nm-thick quantum well of $\mathrm{HgTe}$ (Fig. 3) is close to that obtained for $\mathrm{HgTe} / \mathrm{Hg}_{0.37} \mathrm{Cd}_{0.63} \mathrm{Te}$ superlattices with $L_{w}=3.47 \mathrm{~nm}$ and $L_{b}=7.60 \mathrm{~nm}$. However, the experimental spectra exhibit clear bumps at the step edges, which are absent in calculated ones and can be attributed to excitonic effects.

The quantity $n \mathcal{A}(\hbar \omega)$ measured in InSb/ $\mathrm{Al}_{0.09} \mathrm{In}_{0.91} \mathrm{Sb}$ superlattices at $4.2 \mathrm{~K}$ is also characterized by clear steps at $\pi \alpha$ and $2 \pi \alpha$ (Fig. 9), plus marked peaks which were attributed to excitonic effects [61]. These peaks tend to decrease at higher temperature.

The $\pi \alpha$ quantization is also clearly visible in the case of a $\mathrm{Ga}_{0.47} \mathrm{In}_{0.53} \mathrm{As} / \mathrm{Al}_{0.48} \mathrm{In}_{0.52} \mathrm{As}$ superlattice (Fig. 10), although the excitonic peaks become more prominent.

Figure 11 presents results for GaAs/AlAs superlattices, deduced from measurements made at $2 \mathrm{~K}$ [62]. The average value of $n \mathcal{A}(\hbar \omega)$ is clearly above $\pi \alpha$. Similar values are obtained in GaAs/AlGaAs (not shown) [63]. These values higher than $\pi \alpha$ must be seen as a consequence of strong excitonic effects. However, $\pi \alpha$ remains a good metric of the absorptance.

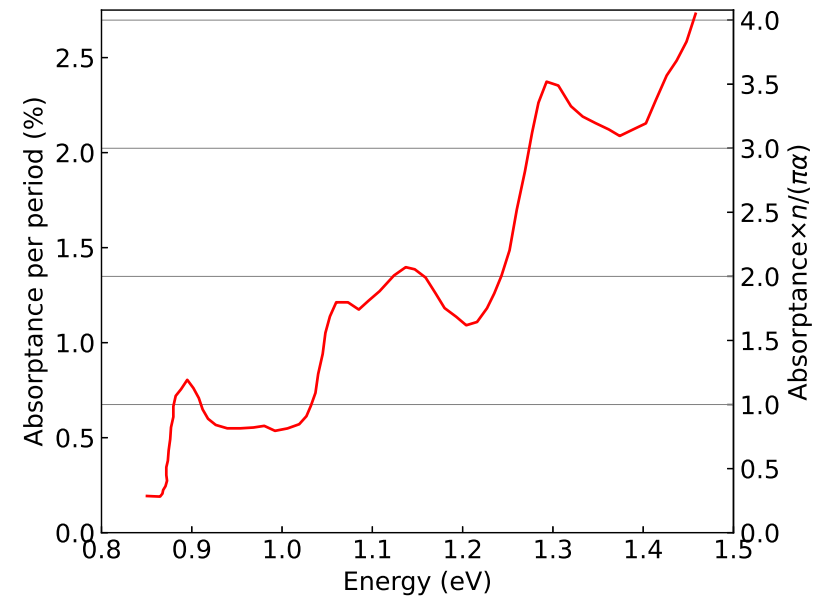

FIG. 10. Absorptance per period in a $\mathrm{Ga}_{0.47} \mathrm{In}_{0.53} \mathrm{As} / \mathrm{Al}_{0.48} \mathrm{In}_{0.52} \mathrm{As}$ superlattice deduced from the measurement [34] of its absorption coefficient (well thickness $=8.5 \mathrm{~nm}$, barrier thickness $=8.5 \mathrm{~nm})$ at $77 \mathrm{~K}($ red line). The right vertical axis gives the absorptance multiplied by the sample refractive index $(n=3.4)$ normalized by $\pi \alpha$.

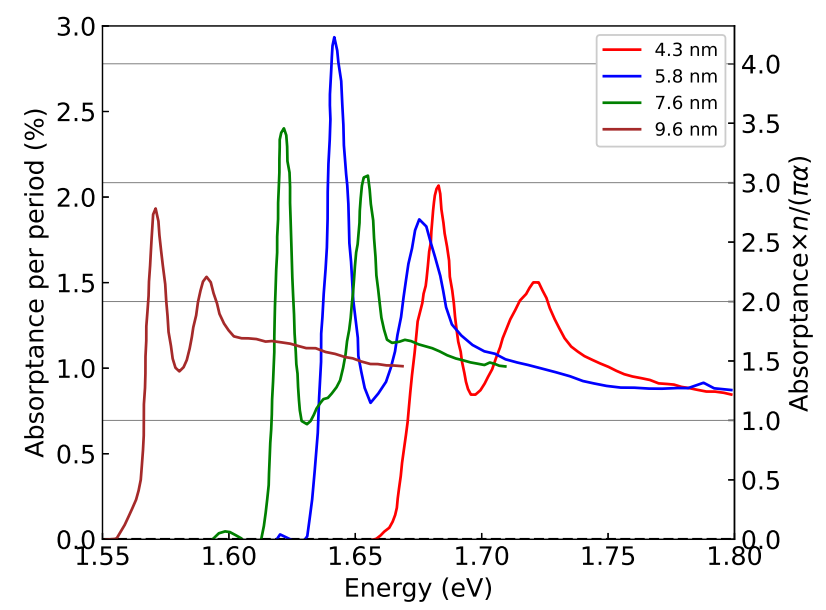

FIG. 11. Absorptance per period in GaAs/AlAs superlattices deduced from the measurement [62] at $2 \mathrm{~K}$ of their absorption coefficient (period $=$ well thickness $L_{w}$ plus barrier thickness $\left.L_{b}\right)$. The right vertical axis gives the absorptance multiplied by the sample refractive index $n$ normalized by $\pi \alpha$. Red line: $L_{w}=4.3 \mathrm{~nm}, L_{b}=6.2 \mathrm{~nm}$. Blue line: $L_{w}=5.8 \mathrm{~nm}, L_{b}=$ $7.1 \mathrm{~nm}$. Green line : $L_{w}=7.6 \mathrm{~nm}, L_{b}=3.3 \mathrm{~nm}$. Brown line : $L_{w}=9.6 \mathrm{~nm}, L_{b}=9.1 \mathrm{~nm}$.

It is important to realize that deviations from $\pi \alpha$ are expected to be stronger in materials characterized by a wide band gap. On the one hand, $P^{2} / E_{g}$ becomes less dominant compared to the other components of the $\mathbf{k} \cdot \mathbf{p}$ Hamiltonian [the $A, L$ and $M$ terms in Eq. (B6) and Eq. (B9) of Appendix B]. Larger deviations are clearly visible in Fig. 3 and Fig. 4 . On the other hand, the dielectric constant is lower, the effective masses are usually higher (especially in the conduction and light-hole bands 

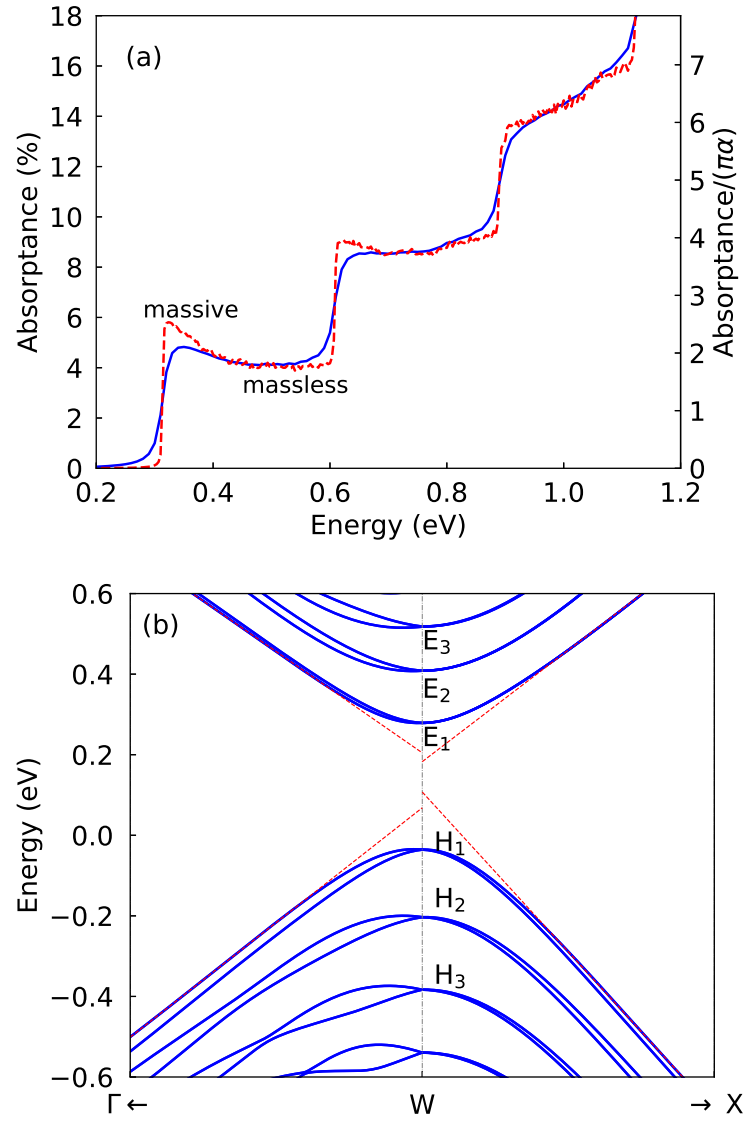

FIG. 12. (a) Bare absorptance $\mathcal{A}_{0}(\hbar \omega)$ calculated for a $6 \mathrm{~nm}$ thick $\mathrm{PbSe}$ quantum wells for two values of the broadening, $\eta=10 \mathrm{meV}$ (blue solid line) or $\eta=1 \mathrm{meV}$ (red dashed line). (b) Zoom on the electron $\left(E_{i}\right)$ and hole $\left(H_{i}\right)$ subbands in the vicinity of the gap. The red dotted lines show a linear variation of the subbands. The full band structure is presented in Fig. S8, Sect. S9 of Supplemental Material [29].

where $\left.1 / m_{e, l h} \propto P^{2} / E_{g}\right)$, which tends to increase the excitonic effects.

\section{QUANTUM WELLS OF PbSe}

By comparison with InAs and other III-V or II-VI compounds, the case of PbSe (and other IV-VI semiconductors with rocksalt lattice) is interesting since the bulk material is characterized in conduction and valence bands by four nonequivalent valleys at the $\mathrm{L}$ point of the Brillouin zone [40]. In absence of experimental data, we have performed calculations of the absorptance for a $6 \mathrm{~nm}$ thick (001) PbSe quantum well. The results, presented in Fig. 12a, show that $\mathcal{A}_{0}(\hbar \omega)$ is also characterized by clear plateaus, but of height $2 \pi \alpha$ instead of $\pi \alpha$.

Figure $12 \mathrm{~b}$ shows the band structure of the quantum well. Due to the quantum confinement, the energy spectrum is characterized by subbands which are (almost) twofold degenerate, fourfold with spin degeneracy (there is small splitting due to inter-valley coupling [40]). In addition, there are two nonequivalent $\mathrm{W}(\pi / a[1,1,0])$ valleys in the Brillouin zone of the $2 \mathrm{D}$ layer. This behavior can be understood by the projection of the four nonequivalent $\mathrm{L}$ points $(\pi / a[1,1,1])$ of the bulk on the $2 \mathrm{D}$ Brillouin zone. The different absorption steps correspond to the transitions allowed only between subbands sharing the same quantum number associated with vertical confinement, i.e., $H_{i} \rightarrow E_{i}$ (Fig. 12b).

Since there are four valleys, absorptance plateaus at $2 \pi \alpha$ mean $\pi \alpha / 2$ contributions from each valley. This behavior is attributed to quasi-linear dispersions of the conduction and valence subbands as one moves away from the band gap (Fig. 12b), for reasons which are discussed in Ref. 40. We then find the situation of graphene where the absorptance per valley is $\pi \alpha / 2$. According to the $\mathbf{k} \cdot \mathbf{p}$ theory, in each valley, subbands $H_{i}$ and $E_{i}$ can be described by a $2 \times 2$ Hamiltonian in which the non-diagonal coupling, of the form $\mathbf{T} \cdot \mathbf{k}$ where $\mathbf{T}$ is a $2 \mathrm{D}$ vector, becomes dominant when moving away from the edges of the subbands. Our tight-binding calculations thus show that the absorptance quantization in PbSe quantum wells has a physical origin much closer to the case of graphene than to the case of III-V or II-VI semiconductors [Fig. 7(b)].

However, the subbands are parabolic in the vicinity of their edges (Fig. 12b). Consequently, if the broadening $\eta$ is reduced, the absorptance spectrum develops peaks just above each optical threshold (Fig. 12a). We then recover the situation of tight-binding models on honeycomb lattices in which a band gap is opened under the effect of SOC or an asymmetry between the two sublattices of the honeycomb (see Sect. S8 in the Supplemental Material [29]). The peaks followed by the plateaus reflect the transformation from massive particles to massless particles [26-28].

\section{CONCLUSION}

In conclusion, we have shown that the quantization of the absorptance in semiconductor quantum wells in units of $\pi \alpha$ is universal. It is predicted for a large number of compounds, provided that the excitonic effects are small. This behavior results from several factors, the main one being the strong coupling of the form $P k_{x}$ and $P k_{y}$, between conduction and valence subbands. Using a minimum model incorporating only these dominant terms in the Hamiltonian, which nevertheless describes the main underlying physics, the absorptance is found to be directly related to $\pi \alpha$, independent of $P$, largely independent of the physical quantities characterizing the semiconductor, as in graphene. The SOC also helps bring the absorptance steps closer to integer values of $\pi \alpha$, redistributing the oscillator strengths. Experimental results obtained on InAs layers, and on superlattices of other materials, support these conclusions. The situation for IV-VI semiconductors such as PbSe is extremely close to that of graphene in the presence of a band gap. We 
believe that this work will provide a simplified view of optical absorption phenomena in $2 \mathrm{D}$ or quasi-2D materials for which excitonic effects are not too strong.

\section{Appendix A: Alternative expression for the absorptance}

\section{General case} form

Using Eq. (3), Eq. (2) can be rewritten in the following

$$
\begin{aligned}
\mathcal{A}_{0}(\hbar \omega)= & \frac{\alpha}{\hbar \omega} \sum_{c, v} \int|\langle c, \mathbf{k}|\nabla H(\mathbf{k})| v, \mathbf{k}\rangle|^{2} \\
& \delta\left(\hbar \omega-E_{c}(\mathbf{k})+E_{v}(\mathbf{k})\right) d^{2} k
\end{aligned}
$$

where we are assuming that, in a cubic semiconductor, the absorptance does not depend on the in-plane orientation of the polarization vector, we can sum the contributions for the two in-plane polarizations and divide by $2\left(|\langle|\nabla H(\mathbf{k}) \cdot \mathbf{x}|\rangle|^{2}+|\langle|\nabla H(\mathbf{k}) \cdot \mathbf{y}|\rangle|^{2}=|\langle|\nabla H(\mathbf{k})|\rangle|^{2}\right)$.

For functions $f$ and $g$ defined over a $n$-dimensional space, we have:

$$
\int_{(n)} f(\mathbf{x}) \delta(g(\mathbf{x})) d \mathbf{x}=\int_{(n-1)} \frac{f(\mathbf{x})}{|\nabla g|} d \sigma(\mathbf{x})
$$

where the second integral is on the $(n-1)$-dimensional surface defined by $g(\mathbf{x})=0$. Equation A1 can be rewritten as

$$
\mathcal{A}_{0}(\hbar \omega)=\frac{\alpha}{\hbar \omega} \sum_{c, v} \int_{\mathcal{L}_{v c}} \frac{|\langle c, \mathbf{k}|\nabla H(\mathbf{k})| v, \mathbf{k}\rangle|^{2}}{\left|\nabla\left(E_{v}(\mathbf{k})-E_{c}(\mathbf{k})\right)\right|} d l
$$

where $\mathcal{L}_{v c}$ is the path in $\mathbf{k}$-space defined by $\hbar \omega-E_{c}(\mathbf{k})+$ $E_{v}(\mathbf{k})=0$.

\section{Isotropic case}

In the case where all terms only depend on $k=|\mathbf{k}|$, Eq. A3 becomes

$$
\mathcal{A}_{0}(\hbar \omega)=\frac{2 \pi \alpha k}{\hbar \omega} \sum_{c, v} \frac{|\langle c, \mathbf{k}|\nabla H(\mathbf{k})| v, \mathbf{k}\rangle|^{2}}{\partial\left|E_{v}(k)-E_{c}(k)\right| / \partial k}
$$

with $k$ such that $E_{c}(k)-E_{v}(k)=\hbar \omega$. This expression can be rewritten in another form given in Eq. (4).

The JDOS per unit area can be calculated in a similar way. For the pair of bands $v$ and $c$, the JDOS is

$$
J_{c, v}(\hbar \omega)=\frac{1}{S} \sum_{\mathbf{k}} \delta\left(\hbar \omega-E_{c}(\mathbf{k})+E_{v}(\mathbf{k})\right)
$$

which, in the isotropic case, transforms into

$$
J_{c, v}(\hbar \omega)=\frac{1}{\pi} \frac{k}{\partial\left|E_{c}(k)-E_{v}(k)\right| / \partial k} .
$$

The bare absorptance can be written as

$$
\mathcal{A}_{0}(\hbar \omega)=\frac{2 \pi^{2} \alpha}{\hbar \omega} \sum_{c, v} J_{c, v}(\hbar \omega)|\langle c, \mathbf{k}|\nabla H(\mathbf{k})| v, \mathbf{k}\rangle|^{2}
$$

with $k$ such that $E_{c}(k)-E_{v}(k)=\stackrel{(\mathrm{A} 7)}{\hbar \omega}$. $|\langle c, \mathbf{k}|\nabla H(\mathbf{k})| v, \mathbf{k}\rangle|^{2} /(\hbar \omega)$ is related to the oscillator strength.

\section{Appendix B: 2D k $p$ model deduced from tight-binding}

Section S3 of the Supplemental Material [29] gives the rationale for describing, in quantum wells, the bands in the vicinity of the band gap with a $2 \mathrm{D} \mathbf{k} \cdot \mathbf{p}$ model. Here we describe how we deduce a $\mathbf{k} \cdot \mathbf{p}$ Hamiltonian directly from tight binding calculations, without adjusting any parameters. We also present calculations of the absorptance in this model, to go beyond the approximations made in the analytic model of Sect. IV B.

\section{Derivation of the $2 \mathrm{D}$ model}

We consider a InAs quantum well of thickness $4.2 \mathrm{~nm}$, chosen because it is characterized by two symmetric surfaces. The system is thus characterized by a perfect inplane square symmetry, which simplifies the derivation of a $2 \mathrm{D} \mathbf{k} \cdot \mathbf{p}$ model. Figure 13 shows that the band structure calculated without SOC is again characterized by many subbands, with complex dispersion in the valence band. However, the absorptance spectrum exhibits a simple behavior (Fig. 5). The analysis of the optical matrix elements shows that the first plateau corresponds to transitions from the two highest valence bands (heavy hole and ligh hole), degenerate at $k_{\|}=0$, to the lowest conduction subband (Fig. S6 of the Supplemental Material [29]).

Next, we deduce a $3 \times 3 \mathbf{k} \cdot \mathbf{p}$ matrix using the calculation of the first-order and second-order derivatives of the tight-binding Hamiltonian matrix with respect to $k_{x}$ and $k_{y}$, restricted to the lowest conduction subband and the two highest valence subbands, but renormalizing the $\mathbf{k} \cdot \mathbf{p}$ parameters to include the effects of the remote bands (see Sect. S5 of the Supplemental Material [29] for the methodology). We obtain

$$
\tilde{H}=\left[\begin{array}{ccc}
E_{c}+A k^{2} & i P k_{x} & i P k_{y} \\
-i P k_{x} & E_{v}+L k_{x}^{2}+M k_{y}^{2} & N k_{x} k_{y} \\
-i P k_{y} & N k_{x} k_{y} & E_{v}+L k_{y}^{2}+M k_{x}^{2}
\end{array}\right]
$$



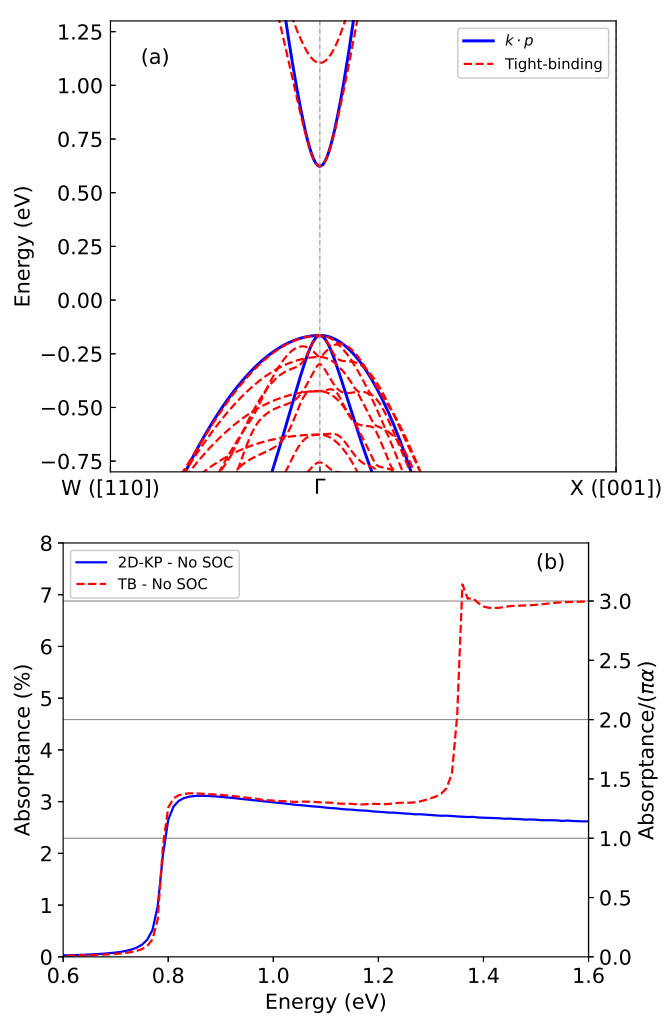

FIG. 13. Comparison between tight-binding (red dashed lines) and 2D k $\cdot \mathbf{p}$ (blue solid lines) calculations for 4.2-nmthick InAs quantum well. SOC is not included. (a) Band structure. (b) Bare absorptance $\mathcal{A}_{0}(\hbar \omega)$.

in the basis of the three vectors $|s\rangle,|x\rangle$ and $|y\rangle$. The parameters directly derived from the tight-binding Hamiltonian are given by (in atomic units): $A=0.00242, P=$ $0.52684, E_{c}=0.02288, E_{v}=-0.00604, L=2.78493$, $M=-0.81699, N=2.71777 . E_{c}$ and $E_{v}$ differ from the bulk values, since they include the effect of the confinement along $z\left(E_{v}=0\right.$ in the bulk).

Figure 13 presents the comparison between tightbinding and $2 \mathrm{D} \mathbf{k} \cdot \mathbf{p}$ band structures, and the comparison between absorptance spectra. In spite of its apparent simplicity, since it only describes two valence subbands of the quantum well, the $2 \mathrm{D} \mathbf{k} \cdot \mathbf{p}$ model gives a perfect description of the first step of $\mathcal{A}_{0}(\hbar \omega)$.

\section{Simplified $2 \mathrm{D} \mathrm{k} \cdot \mathrm{p}$ isotropic model}

In order to obtain analytical expressions for the absorptance, it is useful to derive a simpler $2 \mathrm{D} \mathbf{k} \cdot \mathbf{p}$ isotropic model. We use the two vectors $\left|p_{\|}\right\rangle$and $\left|p_{\perp}\right\rangle$ previously defined [Eq. (9)].

The Hamiltonian matrix element $\left\langle p_{\|}|H| p_{\perp}\right\rangle$ vanishes when $N=L-M$. In the bulk, this takes place when the Luttinger parameters $\gamma_{2}^{L}$ and $\gamma_{3}^{L}$ are equal, which corresponds to the spherical symmetry [64]. In this condition, the Hamiltonian written in $|s\rangle,\left|p_{\|}\right\rangle$and $\left|p_{\perp}\right\rangle$ is simply given by:

$$
H=\left[\begin{array}{ccc}
E_{s}(k) & i P k & 0 \\
-i P k & E_{\|}(k) & 0 \\
0 & 0 & E_{\perp}(k)
\end{array}\right]
$$

with

$$
E_{s}(k)=E_{c}+A k^{2} \quad E_{\|}(k)=E_{v}+L k^{2} \quad E_{\perp}(k)=E_{v}+M k^{2}
$$

The solutions are $\left|\Psi_{h h}\right\rangle=\left|p_{\perp}\right\rangle,\left|\Psi_{e}\right\rangle,\left|\Psi_{l h}\right\rangle$ of energy $E_{h h}(k)$ and

$$
E_{l h}(k)=\left(\frac{E_{s}(k)+E_{\|}(k)}{2}\right) \pm \sqrt{\left(\frac{E_{s}(k)-E_{\|}(k)}{2}\right)^{2}+P^{2} k^{2}},
$$

respectively. $E_{e}(k)$ is the energy of the conduction subband. $E_{h h}(k)$ and $E_{l h}(k)$ are the energies of the heavy and light hole subbands, respectively.

\section{Calculation of the absorptance}

The absorptance $\mathcal{A}_{0}(\hbar \omega)=\mathcal{A}_{h h-e}(\hbar \omega)+\mathcal{A}_{l h-e}(\hbar \omega)$ is calculated analytically using Eq. A4 of Appendix A. In this isotropic approximation, we use the previous parameters for the $2 \mathrm{D} \mathbf{k} \cdot \mathbf{p}$ model except that we set $N=L-M$. After some algebra (Sect. S6 of the Supplemental Material [29]), the different terms can be calculated to the second order in $k$ as

$$
\begin{aligned}
& \mathcal{A}_{h h-e}(\hbar \omega)=\pi \alpha\left(C_{0}+C_{2} k^{2}\right)+O\left(k^{3}\right) \\
& C_{0}=\frac{P^{2}}{\left(A E_{g}-E_{g} M+P^{2}\right)} \\
& C_{2}=-\frac{U}{E_{g}(A-M+U)^{2}} \\
& \times\left(A^{2}+2 A L-4 A M+A U-2 L M+4 L U+3 M^{2}-5 M U\right)
\end{aligned}
$$

with $U=P^{2} / E_{g}$ and $k$ is solution of $E_{e}(k)-E_{h h}(k)=$ $\hbar \omega$. Similarly, we obtain

$$
\begin{aligned}
& \mathcal{A}_{l h-e}(\hbar \omega)=\pi \alpha\left(D_{0}+D_{2} k^{2}\right)+O\left(k^{3}\right) \\
& D_{0}=\frac{P^{2}}{A E_{g}-E_{g} L+2 P^{2}} \\
& D_{2}=-\frac{U\left(5 A^{2}-10 A L+12 A U+5 L^{2}-12 L U+8 U^{2}\right)}{E_{g}(A-L+2 U)^{2}}
\end{aligned}
$$

in which $k$ is solution of $E_{e}(k)-E_{l h}(k)=\hbar \omega$.

With the parameters given above for the 4.2-nm-thick InAs quantum well, $C_{0}=0.92$, which is close to 1 because 
$P^{2} / E_{g} \gg A-M$. Numerical evaluations show that the variation with $k$ and with $\hbar \omega$ is weak (Fig. S4 of the Supplemental Material [29]), i.e., $C_{2} k^{2} \ll C_{0}$. It means that $\mathcal{A}_{h h-e}(\hbar \omega)$ is very close to $\pi \alpha$, but smaller.

With the same parameters, $D_{0}=0.58$ is close to $1 / 2$, because the term $2 P^{2}$ is dominant in the denominator of Eq. B9. $\mathcal{A}_{l h-e}(\hbar \omega)$ is equal to $0.58 \pi \alpha$ at the energy gap but it decreases at higher energy as $D_{2} k^{2}$ becomes non negligible compared to $D_{0}$.
We conclude that the $2 \mathrm{D} \mathbf{k} \cdot \mathbf{p}$ model and its simplified version under isotropic approximation explain very well the absorptance spectrum calculated in tight-binding in absence of SOC. This also completely justifies the simple analytical model of Sect. IV B in which $A, L, M, N=0$.

An additional discussion of compensation mechanisms leading to absorptance quantization is presented in Sect. S6.4 of the Supplemental Material [29].
[1] A. Sommerfeld, The quantum theory of spectral lines, Ann. Phys. 51, 1 (1916).

[2] R. Feynman, QED: The Strange Theory of Light and Matter, Alix G. Mautner memorial lectures (Penguin, 1990).

[3] J. Maciejko, X.-L. Qi, H. D. Drew, and S.-C. Zhang, Topological quantization in units of the fine structure constant, Phys. Rev. Lett. 105, 166803 (2010).

[4] K. v. Klitzing, G. Dorda, and M. Pepper, New method for high-accuracy determination of the fine-structure constant based on quantized hall resistance, Phys. Rev. Lett. 45, 494 (1980).

[5] V. P. Gusynin, S. G. Sharapov, and J. P. Carbotte, Unusual microwave response of dirac quasiparticles in graphene, Phys. Rev. Lett. 96, 256802 (2006).

[6] N. M. R. Peres, F. Guinea, and A. H. Castro Neto, Electronic properties of disordered two-dimensional carbon, Phys. Rev. B 73, 125411 (2006).

[7] L. A. Falkovsky and A. A. Varlamov, Space-time dispersion of graphene conductivity, Eur. Phys. J. B 56, 281 (2007).

[8] R. R. Nair, P. Blake, A. N. Grigorenko, K. S. Novoselov, T. J. Booth, T. Stauber, N. M. R. Peres, and A. K. Geim, Fine structure constant defines visual transparency of graphene, Science 320, 1308 (2008).

[9] K. F. Mak, M. Y. Sfeir, Y. Wu, C. H. Lui, J. A. Misewich, and T. F. Heinz, Measurement of the optical conductivity of graphene, Phys. Rev. Lett. 101, 196405 (2008).

[10] K. F. Mak, L. Ju, F. Wang, and T. F. Heinz, Optical spectroscopy of graphene: From the far infrared to the ultraviolet, Solid State Commun. 152, 1341 (2012).

[11] G. Zhang, S. Huang, F. Wang, Q. Xing, C. Song, C. Wang, Y. Lei, M. Huang, and H. Yan, The optical conductivity of few-layer black phosphorus by infrared spectroscopy, Nat. Commun. 11, 10.1038/s41467-020-156997 (2020)

[12] V. G. Kravets, F. Schedin, and A. N. Grigorenko, Fine structure constant and quantized optical transparency of plasmonic nanoarrays, Nat. Commun. 3, 10.1038/ncomms1649 (2012).

[13] H. Fang, H. A. Bechtel, E. Plis, M. C. Martin, S. Krishna, E. Yablonovitch, and A. Javey, Quantum of optical absorption in two-dimensional semiconductors, Proc. Nat. Acad. Sci. USA 110, 11688 (2013).

[14] K. F. Mak, C. Lee, J. Hone, J. Shan, and T. F. Heinz, Atomically thin $\operatorname{mos}_{2}$ : A new direct-gap semiconductor, Phys. Rev. Lett. 105, 136805 (2010).

[15] M. Bernardi, M. Palummo, and J. C. Grossman, Extraordinary sunlight absorption and one nanometer thick photovoltaics using two-dimensional monolayer materi- als, Nano Letters 13, 3664 (2013).

[16] L. Britnell, R. M. Ribeiro, A. Eckmann, R. Jalil, B. D. Belle, A. Mishchenko, Y.-J. Kim, R. V. Gorbachev, T. Georgiou, S. V. Morozov, A. N. Grigorenko, A. K. Geim, C. Casiraghi, A. H. C. Neto, and K. S. Novoselov, Strong light-matter interactions in heterostructures of atomically thin films, Science 340, 1311 (2013).

[17] K. He, N. Kumar, L. Zhao, Z. Wang, K. F. Mak, H. Zhao, and J. Shan, Tightly bound excitons in monolayer $\mathrm{wse}_{2}$, Phys. Rev. Lett. 113, 026803 (2014).

[18] Y. Li, A. Chernikov, X. Zhang, A. Rigosi, H. M. Hill, A. M. van der Zande, D. A. Chenet, E.-M. Shih, J. Hone, and T. F. Heinz, Measurement of the optical dielectric function of monolayer transition-metal dichalcogenides: $\operatorname{mos}_{2}, \mathrm{MoSe}_{2}, \mathrm{ws}_{2}$, and $\mathrm{WSe}_{2}$, Phys. Rev. B 90, 205422 (2014).

[19] G. Wang, A. Chernikov, M. M. Glazov, T. F. Heinz, X. Marie, T. Amand, and B. Urbaszek, Colloquium: Excitons in atomically thin transition metal dichalcogenides, Rev. Mod. Phys. 90, 021001 (2018).

[20] S. Ithurria, M. D. Tessier, B. Mahler, R. P. S. M. Lobo, B. Dubertret, and A. L. Efros, Colloidal nanoplatelets with two-dimensional electronic structure, Nature Mater 10, 936 (2011).

[21] Z. Chen, B. Nadal, B. Mahler, H. Aubin, and B. Dubertret, Quasi-2d colloidal semiconductor nanoplatelets for narrow electroluminescence, Adv. Funct. Mater. 24, 295 (2014).

[22] T. Stauber, N. M. R. Peres, and A. K. Geim, Optical conductivity of graphene in the visible region of the spectrum, Phys. Rev. B 78, 085432 (2008).

[23] T. Szkopek, Optical frequency conductance, susceptance and admittance of quantum wells, IEEE J. Quantum Electron. 47, 500 (2011).

[24] M. Yoshita, K. Kamide, H. Suzuura, and H. Akiyama, Applicability of continuum absorption in semiconductor quantum wells to absolute absorption-strength standards, Appl. Phys. Lett. 101, 032108 (2012).

[25] L. Matthes, O. Pulci, and F. Bechstedt, Massive dirac quasiparticles in the optical absorbance of graphene, silicene, germanene, and tinene, J. Phys. Condens. Matter 25, 395305 (2013).

[26] T. Stauber, D. Noriega-Pérez, and J. Schliemann, Universal absorption of two-dimensional systems, Phys. Rev. B 91, 115407 (2015).

[27] D. J. Merthe and V. V. Kresin, Transparency of graphene and other direct-gap two-dimensional materials, Phys. Rev. B 94, 205439 (2016).

[28] R. Huang, J. Li, Z. Wu, W. Yang, W. Huang, C. Li, and S. Chen, Universal absorption of two-dimensional mate- 
rials within $\mathrm{k}$ p method, Physics Letters A 382, 3035 (2018).

[29] See Supplemental Material at URL for additional results on InAs, $\mathrm{HgTe}$, PbSe, PbS quantum wells, on the justification and the derivation of the $2 \mathrm{D} \mathbf{k} \cdot \mathbf{p}$ model, on detailed derivations of the analytical models, on calculations of the absorptance in tight-binding models on honeycomb lattices.

[30] G. Fishman, Semi-conducteurs: les bases de la théorie K.P (Editions de l'Ecole Polytechnique, Palaiseau, 2010).

[31] P. R. Wallace, The band theory of graphite, Phys. Rev. 71, 622 (1947).

[32] C. L. Kane and E. J. Mele, Quantum spin hall effect in graphene, Phys. Rev. Lett. 95, 226801 (2005).

[33] T. G. Pedersen, Analytic calculation of the optical properties of graphite, Phys. Rev. B 67, 113106 (2003).

[34] G. Bastard, Wave mechanics applied to semiconductor heterostructures (Les Editions de Physique, Les Ulis, France, 1990).

[35] H. A. Washburn, J. R. Sites, and H. H. Wieder, Electronic profile of $\mathrm{n}$-inas on semi-insulating gaas, J. Appl. Phys. 50, 4872 (1979).

[36] I. Vurgaftman, J. R. Meyer, and L. R. Ram-Mohan, Band parameters for iii-v compound semiconductors and their alloys, J. Appl. Phys. 89, 5815 (2001).

[37] G. Mugny, D. Rideau, F. Triozon, Y.-M. Niquet, C. Kriso, F. Pereira, D. Garetto, C. Tavernier, and C. Delerue, Full-zone k · p parametrization for iii-as materials, in 2015 International Conference on Simulation of Semiconductor Processes and Devices (SISPAD) (2015) pp. 28-31.

[38] C. Delerue and M. Lannoo, Nanostructures: Theory and Modeling (Springer, 2004).

[39] M. Dressel and G. Grüner, in Electrodynamics of Solids: Optical Properties of Electrons in Matter (Cambridge University Press, 2002).

[40] G. Allan and C. Delerue, Confinement effects in pbse quantum wells and nanocrystals, Phys. Rev. B 70, 245321 (2004).

[41] T. B. Boykin, G. Klimeck, R. C. Bowen, and F. Oyafuso, Diagonal parameter shifts due to nearest-neighbor displacements in empirical tight-binding theory, Phys. Rev. B 66, 125207 (2002).

[42] M. Dufour, E. Izquierdo, C. Livache, B. Martinez, M. G. Silly, T. Pons, E. Lhuillier, C. Delerue, and S. Ithurria, Doping as a strategy to tune color of $2 \mathrm{~d}$ colloidal nanoplatelets, ACS Appl. Mater. Interfaces 11, 10128 (2019).

[43] Y. Tan, M. Povolotskyi, T. Kubis, Y. He, Z. Jiang, G. Klimeck, and T. B. Boykin, Empirical tight binding parameters for gaas and mgo with explicit basis through dft mapping, J. Comput. Electron. 12, 56 (2013).

[44] T. B. Boykin, G. Klimeck, and F. Oyafuso, Valence band effective-mass expressions in the $\operatorname{sp}^{3} d^{5} s^{*}$ empirical tightbinding model applied to a si and ge parametrization, Phys. Rev. B 69, 115201 (2004).

[45] G. Allan and C. Delerue, Tight-binding calculations of the optical properties of hgte nanocrystals, Phys. Rev. B 86, 165437 (2012).

[46] A. N. Poddubny, M. O. Nestoklon, and S. V. Goupalov, Anomalous suppression of valley splittings in lead salt nanocrystals without inversion center, Phys. Rev. B 86, 035324 (2012).

[47] Y. M. Niquet, D. Rideau, C. Tavernier, H. Jaouen, and
X. Blase, Onsite matrix elements of the tight-binding hamiltonian of a strained crystal: Application to silicon, germanium, and their alloys, Phys. Rev. B 79, 245201 (2009).

[48] M. Graf and P. Vogl, Electromagnetic fields and dielectric response in empirical tight-binding theory, Phys. Rev. B 51, 4940 (1995).

[49] D. Petrovykh, M. Yang, and L. Whitman, Chemical and electronic properties of sulfur-passivated inas surfaces, Surf. Sci. 523, 231 (2003).

[50] M. L. Cohen and T. K. Bergstresser, Band structures and pseudopotential form factors for fourteen semiconductors of the diamond and zinc-blende structures, Phys. Rev. 141, 789 (1966).

[51] S. Murakami, N. Nagaosa, and S.-C. Zhang, Spin-hall insulator, Phys. Rev. Lett. 93, 156804 (2004).

[52] B. A. Bernevig, T. L. Hughes, and S.-C. Zhang, Quantum spin hall effect and topological phase transition in hgte quantum wells, Science 314, 1757 (2006).

[53] M. König, S. Wiedmann, C. Brüne, A. Roth, H. Buhmann, L. W. Molenkamp, X.-L. Qi, and S.-C. Zhang, Quantum spin hall insulator state in hgte quantum wells, Science 318, 766 (2007).

[54] E. Izquierdo, A. Robin, S. Keuleyan, N. Lequeux, E. Lhuillier, and S. Ithurria, Strongly confined hgte $2 \mathrm{~d}$ nanoplatelets as narrow near-infrared emitters, J. Am. Chem. Soc. 138, 10496 (2016).

[55] A. H. Castro Neto, F. Guinea, N. M. R. Peres, K. S. Novoselov, and A. K. Geim, The electronic properties of graphene, Rev. Mod. Phys. 81, 109 (2009).

[56] E. Kane, Energy band structure in p-type germanium and silicon, J. Phys. Chem. Solids 1, 82 (1956).

[57] C. R. Becker, V. Latussek, A. Pfeuffer-Jeschke, G. Landwehr, and L. W. Molenkamp, Band structure and its temperature dependence for type-iii hgte $/ \mathrm{hg}_{1-x} \mathrm{~cd}_{x} \mathrm{Te}$ superlattices and their semimetal constituent, Phys. Rev. B 62, 10353 (2000).

[58] Y. Lansari, J. W. Han, S. Hwang, L. S. Kim, J. W. Cook, J. F. Schetzina, J. N. Schulman, and N. Otsuka, Properties of hgte-hg0.15cd0.85te superlattices grown by molecular-beam epitaxy, J. Vac. Science Technol. B 7, 241 (1989).

[59] C. R. Becker, V. Latussek, H. Heinke, M. M. Regnet, F. Goschenhofer, S. Einfeldt, L. He, E. Bangert, M. M. Kraus, and G. Landwehr, Molecular beam epitaxial growth and characterization of (001) Hg1-xCdxTeHgTe superlattices, in Growth and Characterization of Materials for Infrared Detectors, Vol. 2021, edited by R. E. Longshore and J. W. Baars, International Society for Optics and Photonics (SPIE, 1993) pp. $138-148$.

[60] C. R. Becker, V. Latussek, W. Spahn, F. Goschenhofer, S. Oehling, and G. Landwehr, Molecular beam epitaxial growth and optical properties of (001) HgTe/Hg1xCdxTe superlattices, in Growth and Characterization of Materials for Infrared Detectors II, Vol. 2554, edited by R. E. Longshore, J. W. Baars, A. Kepten, and J. M. Trombetta, International Society for Optics and Photonics (SPIE, 1995) pp. 6-14.

[61] N. Dai, F. Brown, R. E. Doezema, S. J. Chung, and M. B. Santos, Temperature dependence of exciton linewidths in insb quantum wells, Phys. Rev. B 63, 115321 (2001).

[62] Y. Masumoto, M. Matsuura, S. Tarucha, and H. Okamoto, Direct experimental observation of two-dimensional shrinkage of the exciton wave function 
in quantum wells, Phys. Rev. B 32, 4275 (1985).

[63] W. T. Masselink, P. J. Pearah, J. Klem, C. K. Peng, H. Morkoç, G. D. Sanders, and Y.-C. Chang, Absorption coefficients and exciton oscillator strengths in algaas-gaas superlattices, Phys. Rev. B 32, 8027 (1985).

[64] A. Baldereschi and N. O. Lipari, Spherical model of shallow acceptor states in semiconductors, Phys. Rev. B 8, 2697 (1973). 


\title{
Supplemental Material \\ Universality of the optical absorptance quantization in two-dimensional group-IV, III-V, II-VI and IV-VI semiconductors
}

\author{
Michel Lannoo ${ }^{1}$, P. Tim Prins ${ }^{2}$, Zeger Hens ${ }^{3}$, Daniel Vanmaekelbergh ${ }^{1}$, Christophe Delerue $^{4}$ \\ ${ }^{1}$ Aix Marseille Université, CNRS, Université de Toulon, \\ IM2NP UMR 7334, 13397, Marseille, France \\ ${ }^{2}$ Debye Institute for Nanomaterials Science, University of Utrecht, \\ Princetonplein 1, 3584 CC Utrecht, The Netherlands \\ ${ }^{3}$ Physics and Chemistry of Nanostructures (PCN), \\ Ghent University, 9000 Gent, Belgium \\ ${ }^{4}$ Université de Lille, CNRS, Centrale Lille, Université Polytechnique Hauts-de-France, \\ Junia, UMR 8520 - IEMN, F-59000 Lille, France
}

\section{Contents}

S1 Results for a 12.5-nm-thick quantum well of InAs 3

S2 Band structure of a quantum well of HgTe $\quad 4$

S3 Justification of a $2 \mathrm{D} \mathbf{k} \cdot \mathbf{p}$ model from a 3D one 4

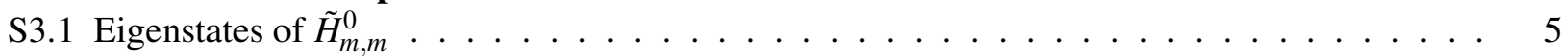

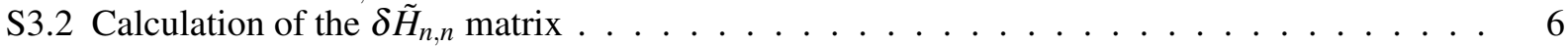

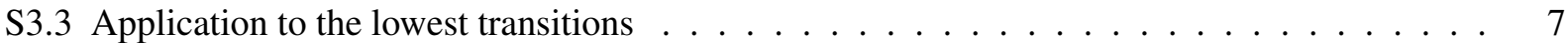

S4 Details on the simple analytical model without SOC $\quad 8$

S4.1 Calculation of the absorptance for the first transition $\Psi_{h h} \rightarrow \Psi_{e} \ldots \ldots \ldots$

S4.2 Calculation of the absorptance for the second transition $\Psi_{l h} \rightarrow \Psi_{e} \ldots \ldots \ldots$

S5 Derivation of a 2D k · p model from tight-binding calculations 9

S6 2D k · p model derived from tight binding 9

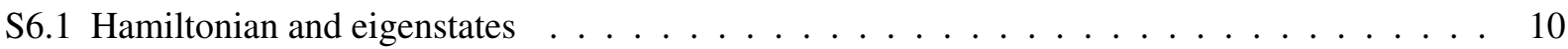

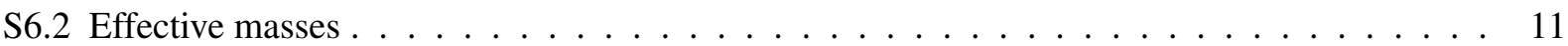

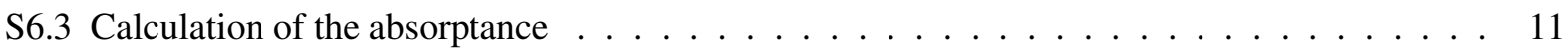

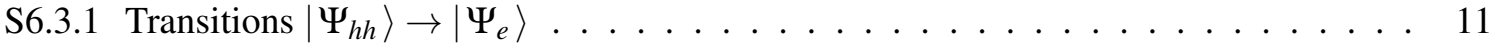

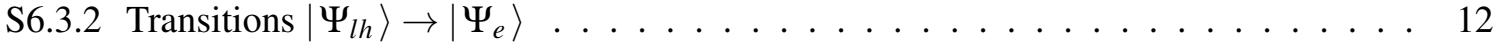

S6.3.3 Comparison with tight-binding calculations . . . . . . . . . . . . . . 12

S6.4 Importance of the term $P^{2} / E_{g}$ on the $\pi \alpha$ quantization $\ldots \ldots \ldots \ldots$ 
S7 Analytical model with SOC 14

S7.1 Details on the analytical calculations . . . . . . . . . . . . . . . . . 14

S7.2 Step heights versus semiconductor characteristics . . . . . . . . . . . . . 15

S7.3 Effect of the SOC from tight binding calculations $\ldots \ldots \ldots \ldots$

S8 Absorptance spectra in tight-binding models on a honeycomb lattice 16

S8.1 Model Hamiltonians . . . . . . . . . . . . . . . . . . . . . . . . 16

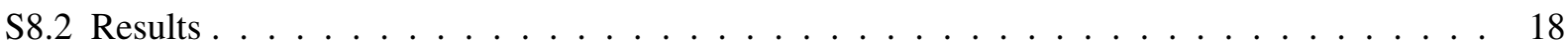

S9 Additional results for quantum wells of rocksalt IV-VI semiconductors 19

S9.1 Band structure of the PbSe quantum well . . . . . . . . . . . . . . . . . . . . . . 19

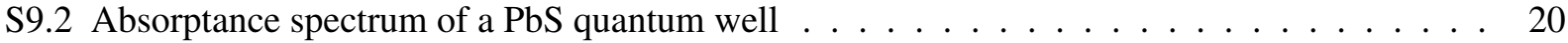



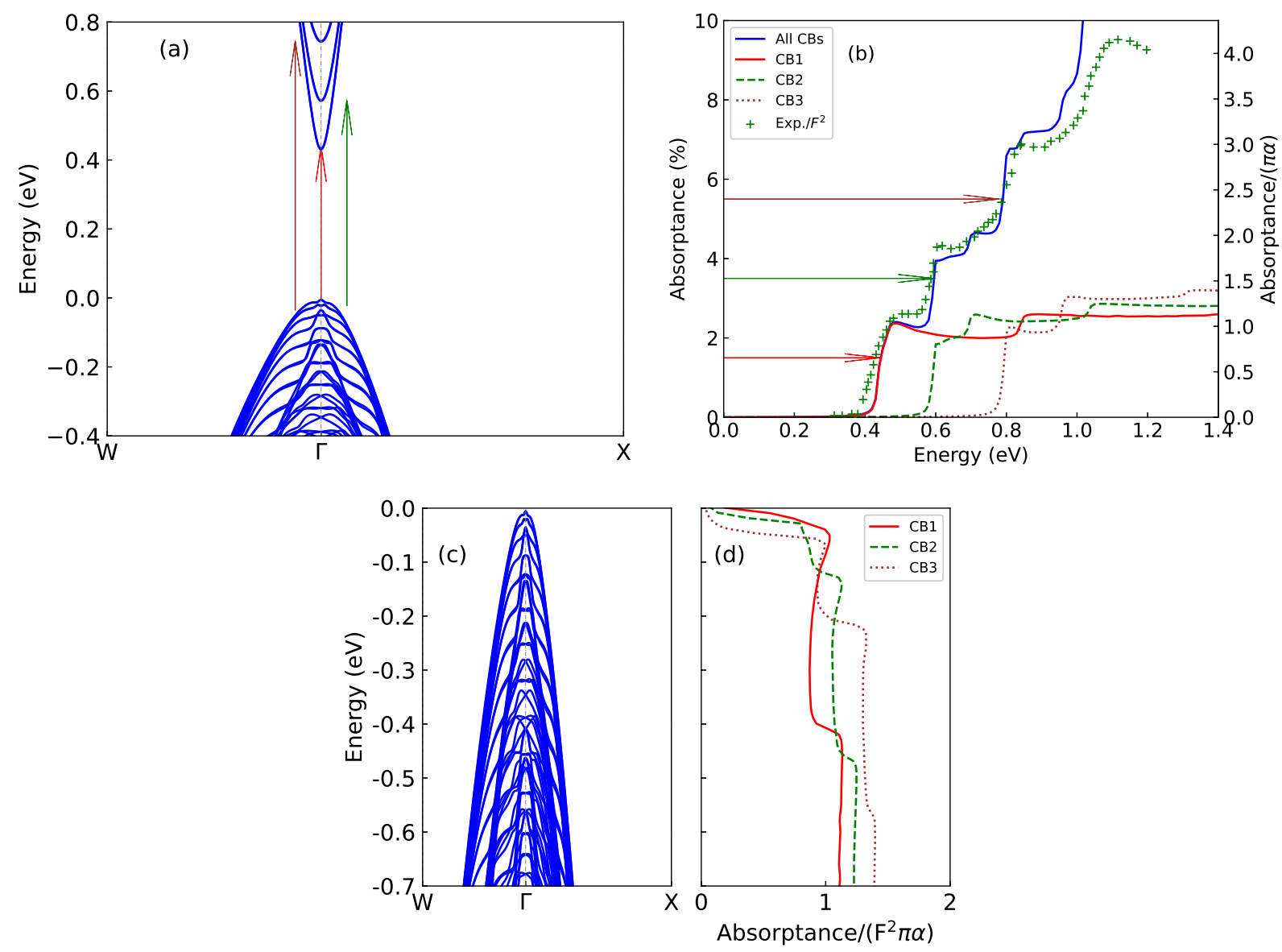

Figure S1: (a) Lowest conduction subbands and highest valence subbands of a $12.5 \mathrm{~nm}$ thick InAs quantum well. (b) Bare absorptance calculated including all conduction subbands (blue solid line), only the first conduction subband (red solid line, CB1), only the second one (green dashed line, CB2) or only the third one (brown dotted line, CB3). Green crosses: experimental data divided by $F^{2}$ for a quantum well thickness of $14 \mathrm{~nm}$ [1]. (c) Zoom on the highest valence subbands and, facing each other, (d) plot of the bare absorptance presented in such a way that each absorptance plateau can be associated to a transition from valence subbands to one of the conduction subbands, the first conduction subband (CB1, red solid line), the second one (CB2, green dashed line), and the third one (CB3, brown dotted line). $\eta=6 \mathrm{meV}$.

\section{S1 Results for a 12.5-nm-thick quantum well of InAs}

Figure S1 present additional results of tight-binding calculations for a thick InAs quantum well (12.5 nm) for which the absorptance was compared to experiments in Fig. 1 of the main document. The different steps at $\approx n \pi \alpha$ shown in Fig. $\mathrm{S} 1 \mathrm{~b}$ can be attributed to transitions $(\mathrm{HH}+\mathrm{LH}+\mathrm{SO})_{i} \rightarrow \mathrm{CB}_{i}$, where $i$ denotes the quantum number related to the vertical confinement effect $(\mathrm{HH}=$ heavy-hole, $\mathrm{LH}=$ light hole, $\mathrm{SO}=$ split-off, $\mathrm{CB}=$ conduction). These results show that the absorptance contributions from the different sets of transitions $(i)$ all behave approximately the same and therefore can be described by the same model. 


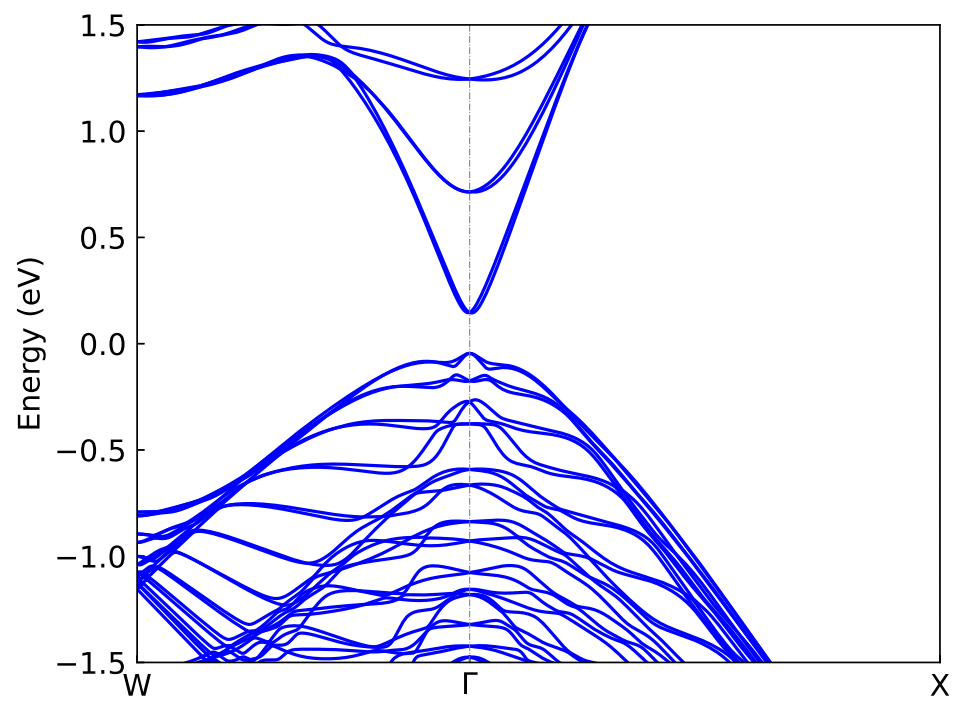

Figure S2: Lowest conduction subbands and highest valence subbands of the HgTe quantum well (thickness of $3.9 \mathrm{~nm}$ ).
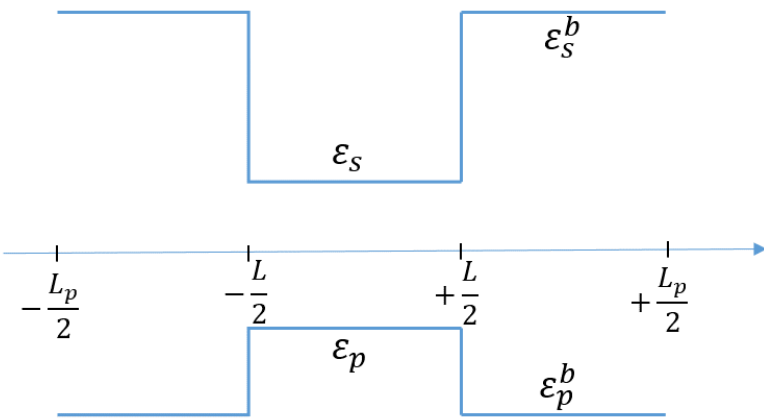

Figure S3: Band diagram for a superlattice along the $z$ direction. We define $\Delta_{s}=\varepsilon_{s}^{b}-\varepsilon_{s}>0$ and $\Delta_{p}=$ $\varepsilon_{p}^{b}-\varepsilon_{p}<0$.

\section{S2 Band structure of a quantum well of HgTe}

The band structure of a HgTe quantum well is presented in Fig. S2. The corresponding absorptance spectrum is shown in Fig. 3 of the main document.

\section{S3 Justification of a 2D $\mathrm{k} \cdot \mathrm{p}$ model from a 3D one}

In this section, we provide a theoretical basis for the use of a $2 \mathrm{D} \mathbf{k} \cdot \mathbf{p}$ Kane model. We consider a periodic system (superlattice) of width $L_{p}$ (Fig. S3) where the quantum well is positioned at the center. We employ the usual 4-band $\mathbf{k} \cdot \mathbf{p}$ hamiltonian without Spin-Orbit Coupling (SOC) [2]. For simplicity, we work in the spherical approximation $(L-M=N)$ but extension to the more general case is straightforward. We expand the envelope function along $z$ into plane waves $\exp \left(i k_{z, n} z\right) / \sqrt{L_{p}}$ where $k_{z, n}=2 n \pi / L_{p}$ due to the periodicity. 
We thus write the hamiltonian into the basis set $u_{l} \exp \left(i \mathbf{k}_{\|} \cdot \mathbf{r}_{\|}\right) \exp \left(i k_{z, n} z\right) / \sqrt{L_{p}}$ where $u_{l}$ are the periodic Bloch functions at $k=0\left[\mathbf{k}_{\|}=\left(k_{x}, k_{y}\right)\right]$. In this basis set, we assume the Kane matrix to be the same all along $z$ except for the discontinuities in conduction $\left[\varepsilon_{s}(z)\right]$ and valence $\left[\varepsilon_{p}(z)\right]$ band edges (Fig. S3). For a given value of $n$, the $4 \times 4$ Kane matrix becomes

$$
\tilde{H}_{n, n}^{0}=\left[\begin{array}{cccc}
\overline{\varepsilon_{s}} & V k_{x} & V k_{y} & V k_{z, n} \\
V^{\dagger} k_{x} & \overline{\varepsilon_{p}}+N k_{x}^{2}+M k_{n}^{2} & N k_{x} k_{y} & N k_{x} k_{z, n} \\
V^{\dagger} k_{y} & N k_{x} k_{y} & \overline{\varepsilon_{p}}+N k_{y}^{2}+M k_{n}^{2} & N k_{y} k_{z, n} \\
V^{\dagger} k_{z, n} & N k_{x} k_{z, n} & N k_{y} k_{z, n} & \overline{\varepsilon_{p}}+N k_{z, n}^{2}+M k_{n}^{2}
\end{array}\right]
$$

where $k_{n}^{2}=k_{x}^{2}+k_{y}^{2}+k_{z, n}^{2}$. $\bar{\varepsilon}_{s}$ and $\overline{\varepsilon_{p}}$ are the average values of $\varepsilon_{s}(z)$ and $\varepsilon_{p}(z)$ over a period.

For a couple $n, m$, the only matrix element is due to the functions $\varepsilon_{s}(z)$ and $\varepsilon_{p}(z)$. We can write

$$
\begin{aligned}
\left(\varepsilon_{s}\right)_{n, m} & =\frac{1}{L_{p}}\left\{\int_{-\frac{L_{p}}{2}}^{\frac{L_{p}}{2}} \varepsilon_{s}^{b} e^{i\left(k_{z, m}-k_{z, n}\right) z} d z+\int_{-\frac{L}{2}}^{\frac{L}{2}}\left(\varepsilon_{s}-\varepsilon_{s}^{b}\right) e^{i\left(k_{z, m}-k_{z, n}\right) z} d z\right\} \\
& =\bar{\varepsilon}_{s} \delta_{n, m}-\left(1-\delta_{n, m}\right) \gamma_{s, n, m}
\end{aligned}
$$

with

$$
\gamma_{s, n, m}=\Delta_{s} \frac{\sin \left[\pi(n-m) L / L_{p}\right]}{\pi(n-m)}
$$

This is also valid for $p$, i.e., $\left(\varepsilon_{p}\right)_{n, m}=\overline{\varepsilon_{p}} \delta_{n, m}-\left(1-\delta_{n, m}\right) \gamma_{p, n, m}$. The $4 \times 4$ coupling matrix between states $n \neq m$ is thus

$$
\tilde{H}_{n, m}^{0}=\left[\begin{array}{cccc}
\gamma_{s, n, m} & 0 & 0 & 0 \\
0 & \gamma_{p, n, m} & 0 & 0 \\
0 & 0 & \gamma_{p, n, m} & 0 \\
0 & 0 & 0 & \gamma_{p, n, m}
\end{array}\right]
$$

In the spirit of self-energy calculations, we write the effective Hamiltonian in the $n, n$ subspace as $\tilde{H}_{n, n}^{0}+$ $\delta \tilde{H}_{n, n}$ with

$$
\delta \tilde{H}_{n, n}=\sum_{m \neq n} \tilde{H}_{n, m}^{0} \tilde{g}_{m, m} \tilde{H}_{m, n}^{0}
$$

where $\tilde{g}_{m, m}$ is the Green's functions $\left(\varepsilon-\tilde{H}_{m, m}^{0}\right)^{-1}$.

\section{S3.1 Eigenstates of $\tilde{H}_{m, m}^{0}$}

Starting from $\tilde{H}_{m, m}^{0}$, this leads to the following equations

$$
\begin{aligned}
& \left(\varepsilon-\overline{\varepsilon_{s}}\right)|s, m\rangle=V k_{m}\left|p_{\|}, m\right\rangle \\
& \left(\varepsilon-\overline{\varepsilon_{p}}\right)|x, m\rangle=V^{\dagger} k_{x}|s, m\rangle+\left(N k_{x}^{2}+M k_{n}^{2}\right)|x, m\rangle+N k_{x}\left(k_{y}|y, m\rangle+k_{z, m}|z, m\rangle\right) \\
& \left(\varepsilon-\overline{\varepsilon_{p}}\right)|y, m\rangle=V^{\dagger} k_{y}|s, m\rangle+\left(N k_{y}^{2}+M k_{n}^{2}\right)|y, m\rangle+N k_{y}\left(k_{x}|x, m\rangle+k_{z, m}|z, m\rangle\right) \\
& \left(\varepsilon-\overline{\varepsilon_{p}}\right)|z, m\rangle=V^{\dagger} k_{z, m}|s, m\rangle+\left(N k_{z, m}^{2}+M k_{n}^{2}\right)|z, m\rangle+N k_{z, m}\left(k_{y}|y, m\rangle+k_{x}|x, m\rangle\right)
\end{aligned}
$$


with

$$
\left|p_{\|}, m\right\rangle=\frac{k_{x}|x, m\rangle+k_{y}|y, m\rangle+k_{z, m}|z, m\rangle}{k_{m}}
$$

From this, we can deduce an equation for $\left|p_{\|}, m\right\rangle$, taking $L=M+N$,

$$
\left(\varepsilon-\overline{\varepsilon_{p}}-L k_{m}^{2}\right)\left|p_{\|}, m\right\rangle=V^{\dagger} k_{m}\left|p_{\|}, m\right\rangle
$$

This, with Eq. (S7), leads to two dispersive bands, for electrons and light holes:

$$
\varepsilon_{m}^{ \pm}=\frac{\overline{\varepsilon_{s}}+\overline{\varepsilon_{p}}+L k_{m}^{2}}{2} \pm \sqrt{\Delta_{m}^{2}+V^{2} k_{m}^{2}}
$$

with $\Delta_{m}=\left(\bar{\varepsilon}_{s}-\overline{\varepsilon_{p}}-L k_{m}^{2}\right) / 2$. The eigenstates are

$$
\begin{aligned}
& |+, m\rangle=c_{s, m}^{+}|s, m\rangle+c_{p_{\|}, m}^{+}\left|p_{\|}, m\right\rangle \\
& |-, m\rangle=c_{p_{\|}, m}^{+*}|s, m\rangle-c_{s, m}^{+}\left|p_{\|}, m\right\rangle
\end{aligned}
$$

with

$$
\begin{aligned}
& c_{s, m}^{+}=\frac{D_{m}}{\sqrt{D_{m}^{2}+1}} \\
& c_{p_{\|}, m}^{+}=\frac{1}{\sqrt{D_{m}^{2}+1}} \frac{V^{\dagger}}{|V|} \\
& D_{m}=\frac{\Delta_{m}+\sqrt{\Delta_{m}^{2}+V^{2} k_{m}^{2}}}{|V| k_{m}}
\end{aligned}
$$

To this one can add the two pure $p$ states, heavy holes, perpendicular to the previous two

$$
\begin{aligned}
& \left|p_{\perp 1}, m\right\rangle=\frac{k_{y}|x, m\rangle-k_{x}|y, m\rangle}{k_{\|}} \\
& \left|p_{\perp 2}, m\right\rangle=\frac{k_{z, m}}{k_{\|}}\left\{\frac{k_{x}|x, m\rangle+k_{y}|y, m\rangle}{k_{m}}-\frac{k_{\|}^{2}}{k_{m} k_{z, m}}|z, m\rangle\right\}
\end{aligned}
$$

with energy

$$
\varepsilon_{\perp 1, m}=\varepsilon_{\perp 2, m}=\varepsilon_{\perp, m}=\bar{\varepsilon}_{p}+M k_{m}^{2}
$$

\section{S3.2 Calculation of the $\delta \tilde{H}_{n, n}$ matrix}

From Eq. (S5) and Eq. (S6) we get

$$
\delta \tilde{H}_{n, n}=\sum_{m \neq n}\left[\begin{array}{cccc}
\gamma_{s, n, m}^{2} g_{s m, s m} & \gamma_{s, n, m} \gamma_{p, n, m} g_{s m, x m} & \gamma_{s, n, m} \gamma_{p, n, m} g_{s m, y m} & \gamma_{s, n, m} \gamma_{p, n, m} g_{s m, z m} \\
\gamma_{s, n, m} \gamma_{p, n, m} g_{x m, s m} & \gamma_{p, n, m}^{2} g_{x m, x m} & \gamma_{p, n, m}^{2} g_{x m, y m} & \gamma_{p, n, m}^{2} g_{x m, z m} \\
\gamma_{s, n, m} \gamma_{p, n, m} g_{y m, s m} & \gamma_{p, n, m}^{2} g_{y m, x m} & \gamma_{p, n, m}^{2} g_{y m, y m} & \gamma_{p, n, m}^{2} g_{y m, z m} \\
\gamma_{s, n, m} \gamma_{p, n, m} g_{z m, s m} & \gamma_{p, n, m}^{2} g_{z m, x m} & \gamma_{p, n, m}^{2} g_{z m, y m} & \gamma_{p, n, m}^{2} g_{z m, z m}
\end{array}\right]
$$


in which the terms $g_{i m, j m}$ are matrix elements of $\tilde{g}_{m, m}$.

The matrix $\delta \tilde{H}_{n, n}$ contains terms like $k_{x}$ or $k_{x} k_{y}$ except for $g_{s m, s m}$. To show this in detail, let us examine the form of the $\tilde{g}_{m, m}$ matrix. We start from the general formula

$$
g_{\alpha m, \beta m}=\sum_{\phi_{m}} \frac{\left\langle\alpha \mid \phi_{m}\right\rangle\left\langle\phi_{m} \mid \beta\right\rangle}{\varepsilon-\varepsilon_{\phi_{m}}}
$$

where $\left|\phi_{m}\right\rangle$ are the eigenstates given by Eq. (S12) and Eq. (S14) of energies given by Eq. (S11) and Eq. (S15). We detail these for each different type of matrix element:

$$
\begin{aligned}
g_{s m, s m}= & \frac{\left|c_{s, m}^{+}\right|^{2}}{\varepsilon-\varepsilon_{m}^{+}}+\frac{\left|c_{s, m}^{-}\right|^{2}}{\varepsilon-\varepsilon_{m}^{-}} \\
g_{s m, x m} & =\left(\frac{c_{s, m}^{+} c_{p_{\|}, m}^{+*}}{\varepsilon-\varepsilon_{m}^{+}}+\frac{c_{s, m}^{-} c_{p_{\|}, m}^{-*}}{\varepsilon-\varepsilon_{m}^{-}}\right) \frac{k_{x}}{k_{m}}=\eta_{s m, p m} \frac{k_{x}}{k_{m}} \quad \text { idem for } k_{x} \text { and } k_{y} \\
g_{x m, y m} & =\left(\frac{\left|c_{p_{\|}, m}^{+}\right|^{2}}{\varepsilon-\varepsilon_{m}^{+}}+\frac{\left|c_{p_{\|}, m}^{-}\right|^{2}}{\varepsilon-\varepsilon_{m}^{-}}-\frac{1}{\varepsilon-\varepsilon_{\perp, m}}\right) \frac{k_{x} k_{y}}{k_{m}^{2}}=\eta_{x m, y m} \frac{k_{x} k_{y}}{k_{m}^{2}} \\
g_{x m, x m} & =\left(\frac{\left|c_{p_{\|}, m}^{+}\right|^{2}}{\varepsilon-\varepsilon_{m}^{+}}+\frac{\left|c_{p_{\|}, m}^{-}\right|^{2}}{\varepsilon-\varepsilon_{m}^{-}}\right) \frac{k_{x}^{2}}{k_{m}^{2}}+\frac{k_{y}^{2}+k_{z, m}^{2}}{k_{m}^{2}\left(\varepsilon-\varepsilon_{\perp, m}\right)}=\eta_{x m, x m}^{(1)} \frac{k_{x}^{2}}{k_{m}^{2}}+\eta_{x m, x m}^{(2)} \frac{k_{y}^{2}+k_{z, m}^{2}}{k_{m}^{2}}
\end{aligned}
$$

idem for permutations of $x, y, z$ with $k_{z}=k_{z, m}$.

Conclusion: This directly shows that $g_{\alpha m, \beta m}$ and thus $\delta \tilde{H}_{n, n}$ has the same form as the Kane matrix and will thus provide renormalized parameters.

\section{S3.3 Application to the lowest transitions}

We start from $\tilde{H}_{0,0}^{0}$ given by Eq. (S1) for which $k_{z, 0}=0$ which splits in a $3 \times 32 \mathrm{D}$ matrix plus one heavy hole $p$ state. The renormalized matrix to consider is thus $\tilde{H}_{0,0}=\tilde{H}_{0,0}^{0}+\delta \tilde{H}_{0,0}$ in which $\delta \tilde{H}_{0,0}$ is given by the sum in Eq. (S16). In that summation, all terms linear in $k_{z, n}$ will vanish since $k_{z,-n}=-k_{z,-n}$. This corresponds to the lowest symmetrical state in the potential well. This gives

$$
\begin{gathered}
\tilde{H}_{n, n}^{0}=\left[\begin{array}{cccc}
\bar{\varepsilon}_{s} & V k_{x} & V k_{y} & 0 \\
V^{\dagger} k_{x} & \overline{\varepsilon_{p}}+L k_{x}^{2}+M k_{y}^{2} & N k_{x} k_{y} & 0 \\
V^{\dagger} k_{y} & N k_{x} k_{y} & \overline{\varepsilon_{p}}+L k_{y}^{2}+M k_{x}^{2} & 0 \\
0 & 0 & 0 & \overline{\varepsilon_{p}}+M k_{\|}^{2}
\end{array}\right]+\sum_{m>0} \\
{\left[\begin{array}{ccccc}
2 \gamma_{s, 0, m}^{2} g_{s m, s m} & 2 \gamma_{s, 0, m} \gamma_{p, 0, m} \eta_{s m, p m} \frac{k_{x}}{k_{m}} & 2 \gamma_{s, 0, m} \gamma_{p, 0, m} \eta_{s m, p m} \frac{k_{y}}{k_{m}} & 0 \\
2 \gamma_{s, 0, m} \gamma_{p, 0, m} \eta_{s m, p m}^{*} \frac{k_{x}}{k_{m}} & 2 \gamma_{p, 0, m}^{2}\left(\eta_{x m, x m}^{(1)} \frac{k_{x}^{2}}{k_{m}^{2}}+\eta_{x m, x m}^{(2)} \frac{k_{y}^{2}+k_{z, m}^{2}}{k_{m}^{2}}\right) & 2 \gamma_{p, 0, m}^{2} \eta_{x m, y m} \frac{k_{x} k_{y}}{k_{m}^{2}} & 0 \\
2 \gamma_{s, 0, m} \gamma_{p, 0, m} \eta_{s m, p m}^{*} \frac{k_{y}}{k_{m}} & 2 \gamma_{p, 0, m}^{2} \eta_{x m, y m}^{*} \frac{k_{x} k_{y}}{k_{m}^{2}} & 2 \gamma_{p, 0, m}^{2}\left(\eta_{x m, x m}^{(1)} \frac{k_{y}^{2}}{k_{m}^{2}}+\eta_{x m, x m}^{(2)} \frac{k_{x}^{2}+k_{z, m}^{2}}{k_{m}^{2}}\right) & 0 \\
0 & 0 & 0 & 2 \gamma_{p, 0, m}^{2} g_{z m, z m}
\end{array}\right]}
\end{gathered}
$$

For optical transitions with polarization vector perpendicular to $O z$ only the $3 \times 3$ submatrix will be involved which justifies the use of a 2D $3 \times 3$ Kane matrix with renormalized parameters. 


\section{S4 Details on the simple analytical model without SOC}

We consider the simplest 2D model described in Section IV.B of the main document. The Hamiltonian is

$$
H=\left[\begin{array}{ccc}
E_{g} & i P k_{x} & i P k_{y} \\
-i P k_{x} & 0 & 0 \\
-i P k_{y} & 0 & 0
\end{array}\right]
$$

where $E_{g}$ is the gap of the quantum well.

\section{S4.1 Calculation of the absorptance for the first transition $\Psi_{h h} \rightarrow \Psi_{e}$}

Transition energy:

$$
E_{e}(k)-E_{h h}(k)=\frac{E_{g}}{2}+\frac{\sqrt{E_{g}^{2}+4 P^{2} k^{2}}}{2}
$$

Optical matrix element:

$$
\left|\left\langle\Psi_{e}|\nabla H(\mathbf{k})| \Psi_{h h}\right\rangle\right|^{2}=\frac{4 P^{4} k^{2}}{4 P^{2} k^{2}+\left(E_{g}-\sqrt{E_{g}^{2}+4 P^{2} k^{2}}\right)^{2}}=\frac{P^{2} \hbar \omega}{2 \hbar \omega-E_{g}}
$$

Derivative of energy:

$$
\frac{1}{k} \frac{\partial\left(E_{e}(k)-E_{h h}(k)\right)}{\partial k}=\frac{2 P^{2}}{\sqrt{E_{g}^{2}+4 P^{2} k^{2}}}
$$

The JDOS including spin degeneracy is (inverse of previous equation $/ \pi$ ):

$$
J_{h h-e}(\hbar \omega)=\frac{2 \hbar \omega-E_{g}}{4 \pi P^{2}}
$$

The denominator of Eq. (4) of the main document is given by

$$
\frac{\partial\left|E_{e}(k)-E_{h h}(k)\right|^{2}}{\partial k^{2}}=\frac{P^{2} \hbar \omega}{\sqrt{\left(E_{g} / 2\right)^{2}+P^{2} k^{2}}}=\frac{2 P^{2} \hbar \omega}{2 \hbar \omega-E_{g}}
$$

\section{S4.2 Calculation of the absorptance for the second transition $\Psi_{l h} \rightarrow \Psi_{e}$}

Transition energy:

$$
\hbar \omega=E_{e}(k)-E_{l h}(k)=2 \sqrt{\frac{E_{g}^{2}}{4}+P^{2} k^{2}}
$$

Optical matrix element:

$$
\left|\left\langle\Psi_{e}|\nabla H| \Psi_{l h}\right\rangle\right|^{2}=\frac{E_{g}^{2} P^{2}}{E_{g}^{2}+4 P^{2} k^{2}}=\left(\frac{E_{g} P}{\hbar \omega}\right)^{2}
$$

Derivative of energy:

$$
\frac{1}{k} \frac{\partial\left(E_{e}(k)-E_{l h}(k)\right)}{\partial k}=\frac{4 P^{2}}{\sqrt{E_{g}^{2}+4 P^{2} k^{2}}}
$$


The JDOS including spin degeneracy is (inverse of previous equation $/ \pi$ ):

$$
J_{l h-e}(\hbar \omega)=\frac{\hbar \omega}{4 \pi P^{2}}
$$

\section{S5 Derivation of a $2 \mathrm{D} \mathrm{k} \cdot \mathrm{p}$ model from tight-binding calculations}

In this section, we present the method used to derive the $\mathbf{k} \cdot \mathbf{p}$ matrix directly from tight-binding calculations for a quantum well. First, the tight-binding Hamiltonian $H$ for the quantum well is written in the basis of Bloch functions as function of $k_{x}$ and $k_{y}$. Second, $H$ is diagonalized at $\mathbf{k}=0$. The eigenvectors form a complete basis of the 2D system. Third, we write $H$ in this basis up to the second order in $\mathbf{k}$,

$$
\begin{aligned}
& H=H^{(0)}+H^{(1)}+H^{(2)} \\
& H^{(1)}=\sum_{i=x, y}\left(\frac{d H}{d k_{i}}\right) k_{i} \\
& H^{(2)}=\frac{1}{2} \sum_{i, j=x, y}\left(\frac{d^{2} H}{d k_{i} d k_{j}}\right) k_{i} k_{j}
\end{aligned}
$$

in which the derivatives are calculated numerically at $\mathbf{k}=0$.

Fourth, we define the subset of the basis in which the $\mathbf{k} \cdot \mathbf{p}$ model will be defined (in our case, the lowest conduction state and the two highest valence states). We represent the states of the subspace by upper case letters, e.g., $I$ of energy $E_{I}$, and the other states of the basis by lower case ones, e.g., $n$ of energy $E_{n}$.

Fifth, we use the Luttinger-Kohn theory of perturbation [3]. If we write $H$ as $\mathscr{H}+V$ where $\mathscr{H}$ contains just the terms restricted to the subspace and $V$ the others, the renormalized Hamiltonian $\tilde{H}$ in the subspace is given to the second order in $V$ by

$$
\tilde{H}_{I J}=\mathscr{H}_{I J}+\frac{1}{2} \sum_{n} V_{I n} V_{n J}\left(\frac{1}{E_{I n}}+\frac{1}{E_{J n}}\right)
$$

If we use for $H$ its expression given in Eq. (S33), the $\mathbf{k} \cdot \mathbf{p}$ Hamiltonian restricted to second order terms in $\mathbf{k}$ is given by

$$
\tilde{H}_{I J}=H_{I J}^{(0)}+H_{I J}^{(1)}+H_{I J}^{(2)}+\frac{1}{2} \sum_{n} H_{I n}^{(1)} H_{n J}^{(1)}\left(\frac{1}{E_{I n}}+\frac{1}{E_{J n}}\right)
$$

Therefore, the computation of the matrices of the first and second derivatives of $H$ allows to completely define the $\mathbf{k} \cdot \mathbf{p}$ Hamiltonian, without adjustable parameters. The last term of Eq. (S35) describes the effect of remote bands that lead to a renormalization of the $\mathbf{k} \cdot \mathbf{p}$ parameters.

\section{S6 2D $\mathrm{k} \cdot \mathrm{p}$ model derived from tight binding}

In this section, we provide details on the calculations presented in Appendix B of the main document. 


\section{S6.1 Hamiltonian and eigenstates}

We give details on 2D k $\cdot \mathbf{p}$ model and its transformation in the isotropic approximation. We have introduced two vectors:

$$
\begin{aligned}
& \left|p_{\|}\right\rangle=\frac{k_{x}|x\rangle+k_{y}|y\rangle}{k} \\
& \left|p_{\perp}\right\rangle=\frac{k_{y}|x\rangle-k_{x}|y\rangle}{k}
\end{aligned}
$$

The matrix element (see definition of $\tilde{H}$ in Eq. (B1) of the main document)

$$
\left\langle p_{\|}|\tilde{H}| p_{\perp}\right\rangle=\frac{k_{x} k_{y}}{k^{2}}\left(k_{x}^{2}-k_{y}^{2}\right)(L-M-N)
$$

vanishes when $N=L-M$. In this condition corresponding to the isotropic approximation, the Hamiltonian written in $|s\rangle,\left|p_{\|}\right\rangle$and $\left|p_{\perp}\right\rangle$ is simply given by:

$$
H=\left[\begin{array}{ccc}
E_{s}(k) & i P k & 0 \\
-i P k & E_{\|}(k) & 0 \\
0 & 0 & E_{\perp}(k)
\end{array}\right]
$$

with

$$
E_{S}(k)=E_{c}+A k^{2} \quad E_{\|}(k)=E_{v}+L k^{2} \quad E_{\perp}(k)=E_{v}+M k^{2}
$$

The solutions are

$$
\begin{aligned}
& \left|\Psi_{e}\right\rangle=\gamma|s\rangle-i \beta\left|p_{\|}\right\rangle \\
& \left|\Psi_{l h}\right\rangle=-i \beta|s\rangle+\gamma\left|p_{\|}\right\rangle \\
& \left|\Psi_{h h}\right\rangle=\left|p_{\perp}\right\rangle
\end{aligned}
$$

with

$$
\begin{aligned}
& \gamma=\frac{P k}{\sqrt{\left(E_{s}(k)-E_{e}(k)\right)^{2}+P^{2} k^{2}}} \\
& \beta=\frac{E_{e}(k)-E_{s}(k)}{\sqrt{\left(E_{s}(k)-E_{e}(k)\right)^{2}+P^{2} k^{2}}}
\end{aligned}
$$

and

$$
E_{l h}(k)=\left(\frac{E_{s}(k)+E_{\|}(k)}{2}\right) \pm \sqrt{\left(\frac{E_{s}(k)-E_{\|}(k)}{2}\right)^{2}+P^{2} k^{2}}
$$




\section{S6.2 Effective masses}

From the previous equations, we can derive the effective masses for electron $\left(m_{e}\right)$, heavy hole $\left(m_{h h}\right)$ and light hole $\left(m_{l h}\right)$ bands. We have:

$$
\begin{aligned}
& \frac{\hbar^{2}}{2 m_{e}}=A+U \\
& \frac{\hbar^{2}}{2 m_{l h}}=-L+U \\
& \frac{\hbar^{2}}{2 m_{h h}}=-M
\end{aligned}
$$

with $U=P^{2} / E_{g}$.

\section{S6.3 Calculation of the absorptance}

The absorption comes from transitions $\left|\Psi_{l h}\right\rangle \rightarrow\left|\Psi_{e}\right\rangle$ and $\left|\Psi_{h h}\right\rangle \rightarrow\left|\Psi_{e}\right\rangle$. The absorptance is calculated analytically using Eq. (A4) of the Appendix A of the main document.

\section{S6.3.1 Transitions $\left|\Psi_{h h}\right\rangle \rightarrow\left|\Psi_{e}\right\rangle$}

We consider $k$ for which $E_{e}(k)-E_{h h}(k)=\hbar \omega$ and we calculate the different contributions to the expression of the absorptance. After some algebra, we obtain:

$$
\begin{gathered}
\left|\left\langle\Psi_{e}|\nabla H(\mathbf{k})| \Psi_{h h}\right\rangle\right|^{2}=(\gamma P+\beta N k)^{2} \\
=\frac{k^{2}\left(2 P^{2}+(L-M)\left(A k^{2}+E_{g}-L k^{2}-\sqrt{4 P^{2} k^{2}+\left(A k^{2}+E_{g}-L k^{2}\right)^{2}}\right)\right)^{2}}{4 P^{2} k^{2}+\left(A k^{2}+E_{g}-L k^{2}-\sqrt{4 P^{2} k^{2}+\left(A k^{2}+E_{g}-L k^{2}\right)^{2}}\right)^{2}} \\
\frac{1}{k} \frac{\partial\left(E_{e}(k)-E_{h h}(k)\right)}{\partial k}=A+L-2 M+\frac{(A-L)\left(\frac{E_{s}(k)-E_{\|}(k)}{2}\right)+P^{2} k^{2}}{\sqrt{\left(\frac{E_{s}(k)-E_{\|}(k)}{2}\right)^{2}+P^{2} k^{2}}} \\
\mathscr{A}_{h h-e}(\hbar \omega)=\frac{2 \pi \alpha k}{E_{e}(k)-E_{h h}(k)} \frac{\left|\left\langle\Psi_{e}|\nabla H(\mathbf{k})| \Psi_{h h}\right\rangle\right|^{2}}{\left|\partial\left(E_{e}(k)-E_{h h}(k)\right) / \partial k\right|}
\end{gathered}
$$

For simplification, it is interesting to perform a second order expansion of the different terms with respect to $k$ :

$$
\begin{gathered}
\left|\left\langle\Psi_{e}|\nabla H(\mathbf{k})| \Psi_{h h}\right\rangle\right|^{2}=-\frac{P^{4} k^{2}}{E_{g}^{2}}+\frac{2 M P^{2} k^{2}}{E_{g}}-\frac{2 L P^{2} k^{2}}{E_{g}}+P^{2}+O\left(k^{3}\right) \\
\frac{1}{k} \frac{\partial\left(E_{e}(k)-E_{h h}(k)\right)}{\partial k}=-\frac{4 P^{4} k^{2}}{E_{g}^{3}}+\frac{4 L P^{2} k^{2}}{E_{g}^{2}}+\frac{2 P^{2}}{E_{g}}-2 M-\frac{4 A P^{2} k^{2}}{E_{g}^{2}}+2 A+O\left(k^{3}\right)
\end{gathered}
$$




$$
E_{e}(k)-E_{h h}(k)=\frac{P^{2} k^{2}}{E_{g}}-M k^{2}+E_{g}+A k^{2}+O\left(k^{3}\right)
$$

We deduce the absorptance to the second order in $k$ :

$$
\begin{aligned}
& \mathscr{A}_{h h-e}(\hbar \omega)=\pi \alpha\left(C_{0}+C_{2} k^{2}\right)+O\left(k^{3}\right) \\
& C_{0}=\frac{U}{(A-M+U)} \\
& C_{2}=-\frac{U\left(A^{2}+2 A L-4 A M+A U-2 L M+4 L U+3 M^{2}-5 M U\right)}{E_{g}(A-M+U)^{2}}
\end{aligned}
$$

S6.3.2 Transitions $\left|\Psi_{l h}\right\rangle \rightarrow\left|\Psi_{e}\right\rangle$

The contribution of $\left|\Psi_{l h}\right\rangle \rightarrow\left|\Psi_{e}\right\rangle$ transitions to the absorptance can be calculated similarly. Using the different contributions

$$
\begin{aligned}
& \hbar \omega=E_{e}(k)-E_{l h}(k)=2 \sqrt{P^{2} k^{2}+\frac{\left(A k^{2}+E_{g}-L k^{2}\right)^{2}}{4}} \\
&\left|\left\langle\Psi_{e}|\nabla H| \Psi_{l h}\right\rangle\right|^{2} \\
&=\left(P\left(2 \gamma^{2}-1\right)-2 \gamma \beta k[A-L]\right)^{2} \\
&= \frac{P^{2}\left(A^{2} k^{4}-2 A E_{g} k^{2}-2 A L k^{4}+E_{g}^{2}+2 E_{g} L k^{2}+L^{2} k^{4}\right)}{A^{2} k^{4}+2 A E_{g} k^{2}-2 A L k^{4}+E_{g}^{2}-2 E_{g} L k^{2}+L^{2} k^{4}+4 P^{2} k^{2}} \\
&= \frac{E_{g}^{2} P^{2}+4 E_{g} P^{2} k^{2}(-A+L)-4 P^{4} k^{2}}{E_{g}^{2}}+O\left(k^{3}\right) \\
& \frac{1}{k} \frac{\partial\left(E_{e}(k)-E_{l h}(k)\right)}{\partial k}=\frac{2\left(2 P^{2}+(A-L)\left(A k^{2}+E_{g}-L k^{2}\right)\right)}{\sqrt{4 P^{2} k^{2}+\left(A k^{2}+E_{g}-L k^{2}\right)^{2}}}
\end{aligned}
$$

we obtain $\mathscr{A}_{l h-e}(\hbar \omega)$ to the second order in $k$ :

$$
\begin{aligned}
& \mathscr{A}_{l h-e}(\hbar \omega)=\pi \alpha \times\left(D_{0}+D_{2} k^{2}\right) \\
& D_{0}=\frac{U}{A-L+2 U} \\
& D_{2}=-\frac{U\left(5 A^{2}-10 A L+12 A U+5 L^{2}-12 L U+8 U^{2}\right)}{E_{g}(A-L+2 U)^{2}}
\end{aligned}
$$

\section{S6.3.3 Comparison with tight-binding calculations}

Figure S4 shows that the total absorptance $\mathscr{A}_{0}(\hbar \omega)$ calculated under the isotropic approximation is in excellent agreement with $\mathscr{A}_{0}(\hbar \omega)$ calculated numerically using the $2 \mathrm{D} \mathbf{k} \cdot \mathbf{p}$ model originally deduced from the tight-binding Hamiltonian, which itself is in excellent agreement with tight-binding calculations. It demonstrates the validity of the isotropic approximation. 


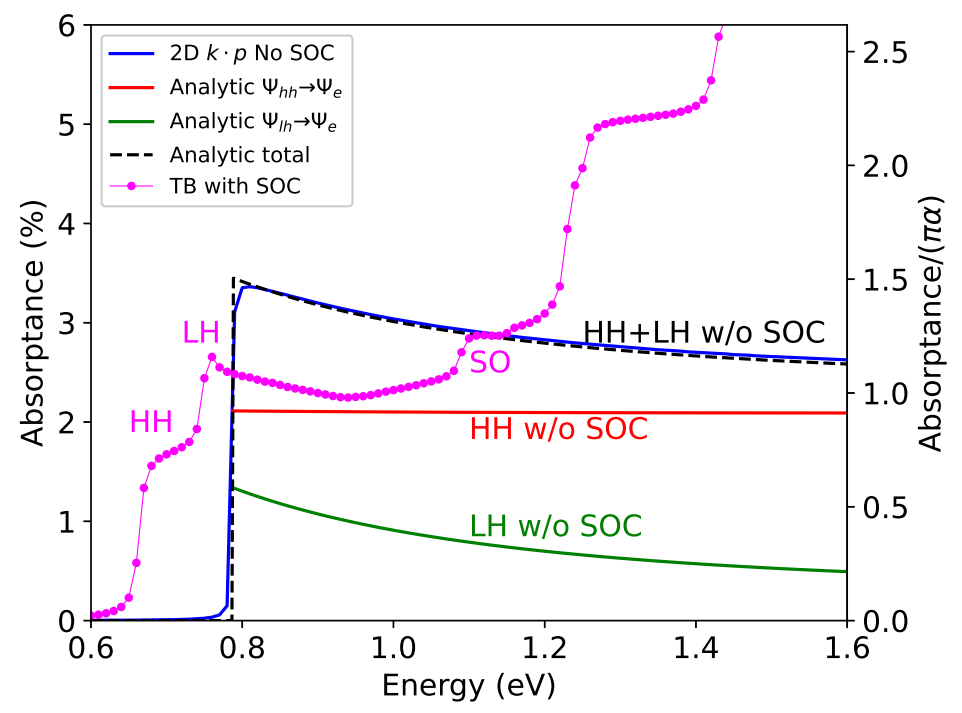

Figure S4: Comparison between different calculations of the absorptance spectrum for a 4.2-nm-thick InAs quantum well. Blue solid line: 2D k · p model (no SOC) directly deduced from the tight-binding Hamiltonian, calculated using a fully numerical approach $(\eta=1 \mathrm{meV})$. Black dashed line: Same but with the analytical $2 \mathrm{D} \mathrm{k} \cdot \mathbf{p}$ model under isotropic approximation. The contributions coming from $\left|\Psi_{h h}\right\rangle \rightarrow\left|\Psi_{e}\right\rangle(\mathrm{HH} \rightarrow \mathrm{CB})$ and $\left|\Psi_{l h}\right\rangle \rightarrow\left|\Psi_{e}\right\rangle(\mathrm{LH} \rightarrow \mathrm{CB})$ transitions are shown with red and green solid lines, respectively. Magenta dotted solid line: Tight-binding calculations with SOC.

\section{S6.4 Importance of the term $P^{2} / E_{g}$ on the $\pi \alpha$ quantization}

Equations (S56) and (S62) show that $C_{0} \approx 1$ and $D_{0} \approx 1 / 2$ when $P^{2} / E_{g} \gg A,|L|,|M|$, which leads to the $\pi \alpha$ quantization of the absorptance after inclusion of the spin-orbit coupling. However, Eq. (S48) shows that we could have the same effective masses with a much lower value of $U=P^{2} / E_{g}$, compensating via $A$ and $L$. In that case, the effective masses would be mainly determined by the couplings with the bands outside the subspace. Interestingly, $C_{0}$ and $D_{0}$ can be rewritten as

$$
\begin{aligned}
& C_{0}=\frac{U}{\frac{\hbar^{2}}{2}\left(\frac{1}{m_{e}}+\frac{1}{m_{h h}}\right)} \\
& D_{0}=\frac{U}{\frac{\hbar^{2}}{2}\left(\frac{1}{m_{e}}+\frac{1}{m_{l h}}\right)}
\end{aligned}
$$

A compensation between numerator and denominator is done when $U \gg A,|L|,|M|$, i.e., when $\hbar^{2} /\left(2 m_{e}\right)$ and $\hbar^{2} /\left(2 m_{l h}\right)$ are approximately equal to $U$ [Eq. (S48)]. But, if we decrease $U$ while keeping the effective masses constant, $C_{0}$ and $D_{0}$ become very small compared to 1 and $1 / 2$, the absorptance is then no longer quantized in units of $\pi \alpha$, but can take any (smaller) value depending on $U$.

Therefore, we deduce that the compensation in $\mathscr{A}_{0}(\hbar \omega)$ between the joint density of states and the optical matrix elements comes mainly from the combination of three effects. First, the dispersion of the electron and light-hole bands is mainly governed by couplings of type $P k$ between them. Second, $P$ also determines the optical matrix elements between valence and conduction bands. Third, the valence band whose dispersion 
does not depend on the $P k$ coupling is characterized by a heavy effective mass $\left(M \ll U, m_{e} \ll m_{h h}\right)$.

Since $U$ is equal to $P^{2} / E_{g}$, and $P$ is known to be rather constant in different types of III-V and II-VI semiconductors, we expect that the absorptance quantization will be less accurate in semiconductors with a large gap $E_{g}$. This is what we found with tight-binding calculations (see main document).

\section{S7 Analytical model with SOC}

\section{S7.1 Details on the analytical calculations}

As discussed in Sect. IV.C of the main document, the Kane Hamiltonian is written in the basis of the eigenstates of $\mathbf{J}^{2}$ and $J_{Z}$ where $Z$ is defined along the $\mathbf{k}$ vector, i.e., $|Z \uparrow\rangle \propto k_{x}|x \uparrow\rangle+k_{y}|y \uparrow\rangle+k_{z}|z \uparrow\rangle$ (idem for $\downarrow)$.

In the case of a quantum well, we reduce it to a $2 \mathrm{D}$ problem with $k_{z}=0\left(k=k_{\|}\right)$, i.e.,

$$
|Z \uparrow\rangle=\frac{k_{x}|x \uparrow\rangle+k_{y}|y \uparrow\rangle}{k_{\|}} .
$$

We define the two orthogonal states

$$
\begin{aligned}
& |X \uparrow\rangle=\frac{k_{y}|x \uparrow\rangle-k_{x}|y \uparrow\rangle}{k_{\|}}, \\
& |Y \uparrow\rangle=|z \uparrow\rangle,
\end{aligned}
$$

with similar expressions for spin down states.

The eigenstates of $\mathbf{J}^{2}$ and $J_{Z}$ are given by [4]

$$
\begin{aligned}
& \left|\frac{3}{2}, \frac{1}{2}\right\rangle=-\sqrt{\frac{2}{3}}|Z \uparrow\rangle+\frac{1}{\sqrt{6}}|X+i Y, \downarrow\rangle \\
& \left|\frac{1}{2}, \frac{1}{2}\right\rangle=\frac{1}{\sqrt{3}}|Z \uparrow\rangle+\frac{1}{\sqrt{3}}|X+i Y, \downarrow\rangle \\
& \left|\frac{3}{2},-\frac{1}{2}\right\rangle=-\sqrt{\frac{2}{3}}|Z \downarrow\rangle-\frac{1}{\sqrt{6}}|X-i Y, \uparrow\rangle \\
& \left|\frac{1}{2},-\frac{1}{2}\right\rangle=\frac{1}{\sqrt{3}}|Z \downarrow\rangle-\frac{1}{\sqrt{3}}|X-i Y, \uparrow\rangle \\
& \left|\frac{3}{2}, \frac{3}{2}\right\rangle=\frac{1}{\sqrt{2}}|X+i Y, \uparrow\rangle \\
& \left|\frac{3}{2},-\frac{3}{2}\right\rangle=\frac{1}{\sqrt{2}}|X-i Y, \downarrow\rangle .
\end{aligned}
$$

The Hamiltonian is given by two blocks like Eq. (19) of the main document.

By treating $P k$ within second order perturbation theory, the energy dispersion with respect to $k=0$ is given by 


$$
\begin{aligned}
& \varepsilon_{e}(k)=\varepsilon_{g}+\frac{2}{3} \frac{P^{2} k^{2}}{\varepsilon_{g}}+\frac{1}{3} \frac{P^{2} k^{2}}{\varepsilon_{g}+\Delta} \\
& \varepsilon_{h h}(k)=0 \\
& \varepsilon_{l h}(k)=-\frac{2}{3} \frac{P^{2} k^{2}}{\varepsilon_{g}} \\
& \varepsilon_{s o}(k)=-\Delta-\frac{1}{3} \frac{P^{2} k^{2}}{\varepsilon_{g}+\Delta} .
\end{aligned}
$$

This gives at $k=0$

$$
\begin{aligned}
& \frac{\partial\left(\varepsilon_{e}(k)-\varepsilon_{l h}(k)\right)^{2}}{\partial k^{2}}=\frac{8 P^{2}}{3}\left(1+\frac{\varepsilon_{g}}{4\left(\varepsilon_{g}+\Delta\right)}\right), \\
& \frac{\partial\left(\varepsilon_{e}(k)-\varepsilon_{s o}(k)\right)^{2}}{\partial k^{2}}=\frac{4 P^{2}}{3}\left(1+\frac{\varepsilon_{g}+\Delta}{\varepsilon_{g}}\right), \\
& \frac{\partial\left(\varepsilon_{e}(k)-\varepsilon_{h h}(k)\right)^{2}}{\partial k^{2}}=\frac{4 P^{2}}{3}\left(1+\frac{\varepsilon_{g}}{2\left(\varepsilon_{g}+\Delta\right)}\right) .
\end{aligned}
$$

The optical matrix elements at $k=0$ are:

$$
\begin{gathered}
\left|\left\langle s \uparrow|\nabla H(\mathbf{k})| \frac{3}{2}, \frac{1}{2}\right\rangle\right|^{2}=\frac{2 P^{2}}{3}, \\
\left|\left\langle s \uparrow|\nabla H(\mathbf{k})| \frac{1}{2}, \frac{1}{2}\right\rangle\right|^{2}=\frac{P^{2}}{3}, \\
\left|\left\langle s \uparrow|\nabla H(\mathbf{k})| \frac{3}{2}, \frac{3}{2}\right\rangle\right|^{2}=\frac{P^{2}}{2} .
\end{gathered}
$$

From this, we could calculate the absorptance using Eq. (4) of the main document.

\section{S7.2 Step heights versus semiconductor characteristics}

Figure S5 presents the step height calculated using Eq. (20) $\left[\mathscr{A}_{0}\left(\varepsilon_{g}\right)\right]$ and Eq. (21) $\left[\delta \mathscr{A}_{0}\left(\varepsilon_{g}+\Delta\right)\right]$ of the main document, versus $x=\varepsilon_{g} / \Delta$.

The development in series with respect to $x=1$ of Eq. (20) of the main document gives

$$
\pi \alpha\left\{1.225-0.341 x+0.241 x^{2}-0.098 x^{3}+0.017 x^{4}+O\left((x-1)^{5} ; x \rightarrow 1\right)\right\} .
$$

The term in braces is close to unity. $\mathscr{A}_{0}\left(\varepsilon_{g}\right)$ remains close to $\pi \alpha$, within $\pm 10 \%$ for $x$ varying from 0.5 to 5.

\section{S7.3 Effect of the SOC from tight binding calculations}

Figure S6 presents the variation of the valence subband energies and optical matrix elements at $k=0$ when we vary the strength of the SOC. First, the SOC splits the two highest degenerate energy subbands. Second, 


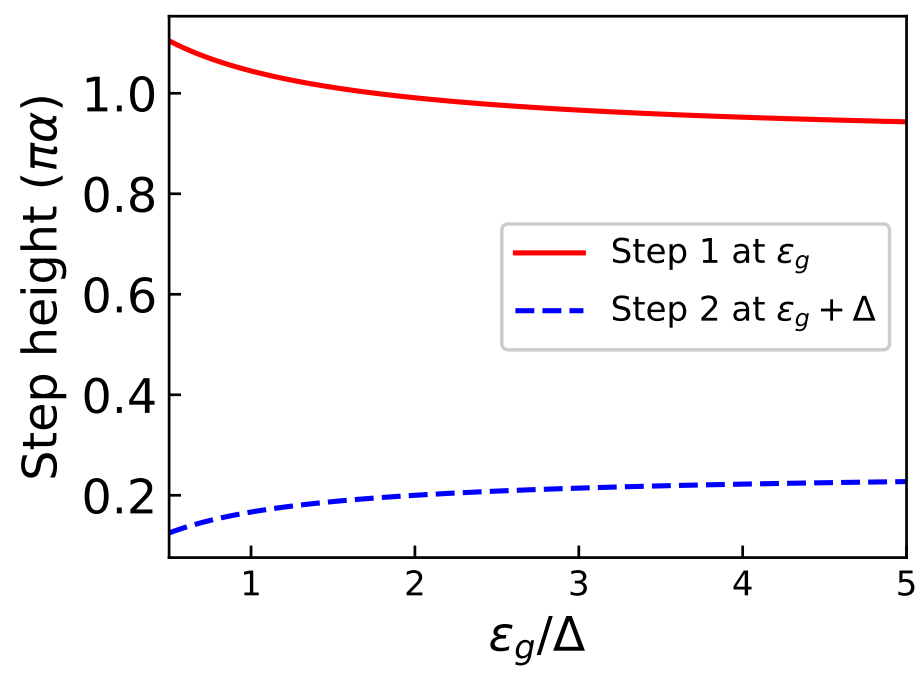

Figure S5: Height of the steps in unit of $\pi \alpha$ calculated using the analytical model including SOC versus $\varepsilon_{g} / \Delta$ (b). Red solid line: first step, $\mathscr{A}_{0}\left(\varepsilon_{g}\right)$. Blue dashed line: second step, $\delta \mathscr{A}_{0}\left(\varepsilon_{g}+\Delta\right)$.

it couples the lowest of these two subbands to a third subband located at lower energy $(\sim-0.3 \mathrm{eV})$, which leads to a redistribution of the optical oscillator strengths, their sum remaining approximately constant. At increasing SOC, this leads to the conventional picture of three valence subbands derived from heavy-hole, light-hole, and split-off bands. [4]

\section{S8 Absorptance spectra in tight-binding models on a honeycomb lattice}

\section{S8.1 Model Hamiltonians}

As already demonstrated in Ref. [5], the case of Hamiltonians on the honeycomb lattice is particularly interesting since it allows to study the effect on the absorptance spectrum of the transition from massless particles to massive ones. In this appendix, we illustrate different physical effects that induce the opening of a band gap at the Dirac point of a graphene band structure. These effects are, the asymmetry between sublattices A and B of the honeycomb, the intrinsic Spin-Orbit Coupling (SOC), and the Rashba SOC. We start from the tight-binding model that describes the $\pi$ bands of graphene [6], with one orbital on each lattice site (two including spin). The Hamiltonian is written as $H=H_{0}+H_{\mathrm{NN}}+H_{\mathrm{ISO}}+H_{\mathrm{RSO}}$. Here, $H_{0}$ incorporates the on-site energies $E_{A}$ and $E_{B}$ on sublattice $\mathrm{A}$ and $\mathrm{B}$, respectively. The term

$$
H_{\mathrm{NN}}=t \sum_{\langle i, j\rangle} \sum_{\alpha} c_{i, \alpha}^{\dagger} c_{j, \alpha}
$$

includes hopping terms, where $\langle i, j\rangle$ denotes nearest-neighbor (NN) sites, $\alpha$ denotes spin, and $t=-2.8 \mathrm{eV}$ is the hopping parameter typically used for graphene [7].

The intrinsic SOC term $H_{\text {ISO }}$ couples the electron orbital momentum $\mathbf{L}$ and spin $\mathbf{S}$. There is no on-site term because the orbital momentum is frozen. However, as shown by Kane and Mele [8], the intrinsic SOC can introduce a next-nearest-neighbour (NNN) hopping term, which is written as 


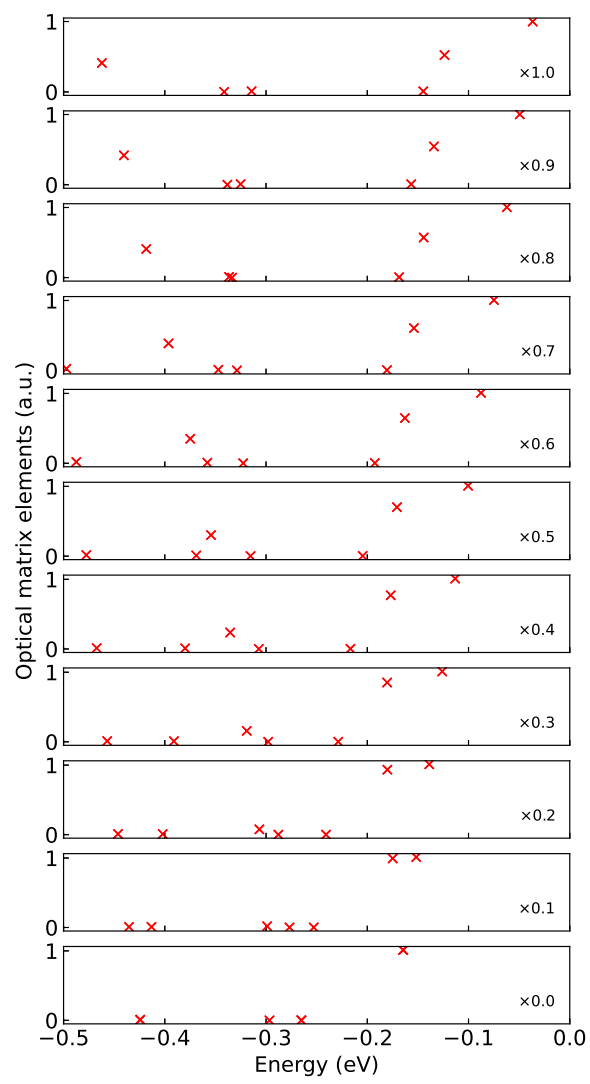

Figure S6: Evolution of the energy levels in a 4.2-nm-thick InAs layer, at $k=0$ in the valence band when the SOC is scaled by a factor varying from 0.0 to 1.0. The vertical axis indicates the optical matrix element $|\langle c, \mathbf{0}|\nabla H(\mathbf{k})| v, \mathbf{0}\rangle|^{2}$ for the transition to the lowest conduction subband (same scale for all figures).

$$
H_{\mathrm{ISO}}=i \lambda \sum_{\langle\langle i, j\rangle\rangle} \sum_{\alpha} c_{i, \alpha}^{\dagger} S_{\alpha \alpha}^{z} v_{i j} c_{j, \alpha}
$$

Here, the summation is over NNNs, and $v_{i j}= \pm 1$, with the sign depending on the outer product of the two NN vectors that connect sites $i$ and $j$. The Rashba SOC term is written as a NN-hopping term

$$
H_{\mathrm{RSO}}=i \gamma \sum_{\langle i, j\rangle} \sum_{\alpha, \beta} c_{i, \alpha}^{\dagger}\left[\hat{z} \cdot\left(\mathbf{S} \times \mathbf{r}_{i j}\right)\right]_{\alpha \beta} c_{j, \beta}
$$

$\lambda$ and $\gamma$ are coupling constants that we adjust to open a visible gap. In any case, we set the Fermi level at the zero of energy, i.e., at the Dirac point of graphene. 

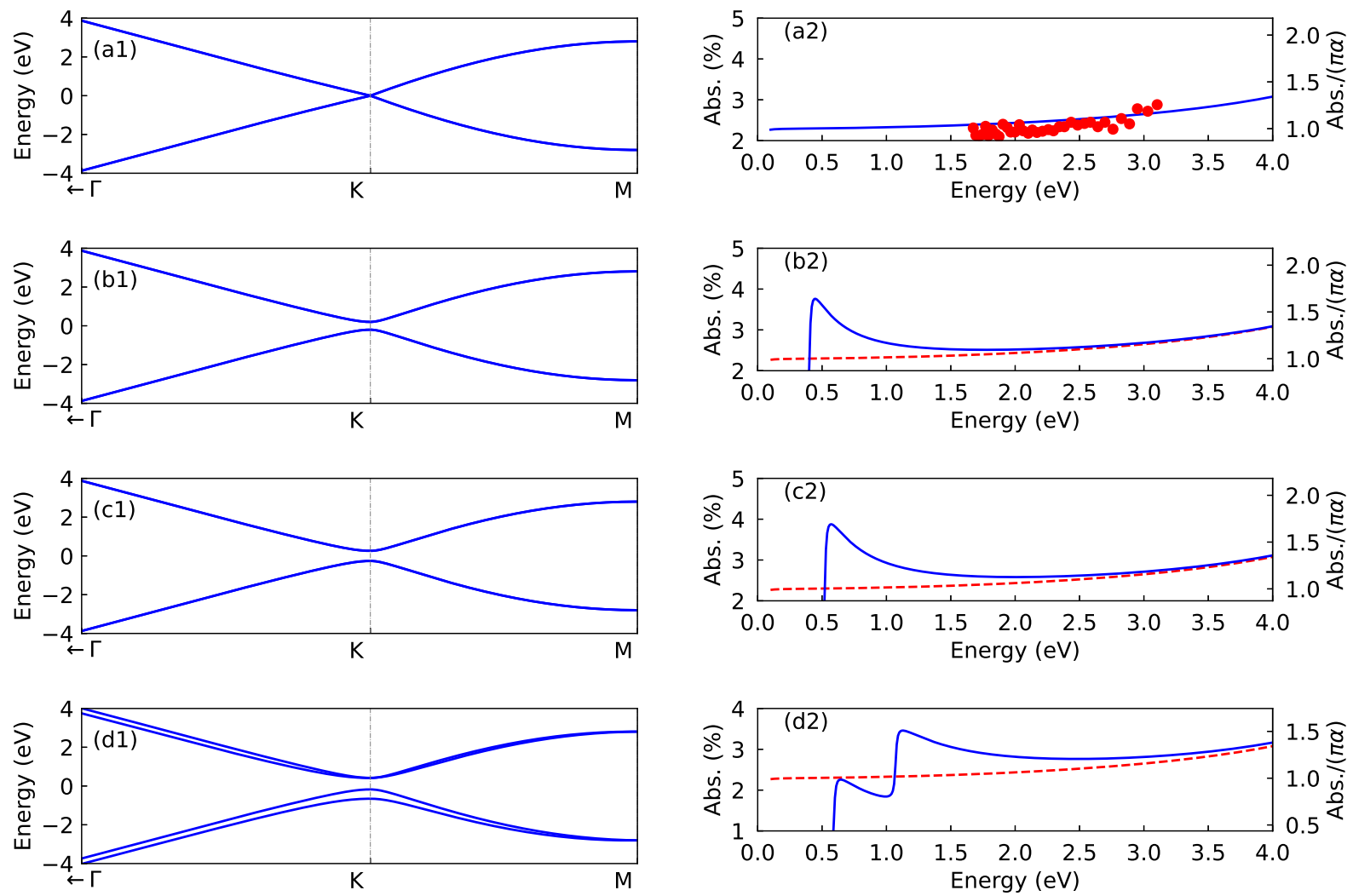

Figure S7: Band structures (x1) and absorptance spectra (x2) calculated in the following configurations. (a1,a2) Model of graphene, no lattice asymmetry $\left(E_{A}=E_{B}=0\right)$, and no SOC. The red points represent the experimental data of Ref. [9]. (b1,b2) Same but with a sublattice asymmetry, $E_{A}=0.2 \mathrm{eV}$ and $E_{B}=-0.2 \mathrm{eV}$. (c1,c2) No asymmetry $\left(E_{A}=E_{B}=0\right)$ but intrinsic SOC $(\lambda=0.1 \mathrm{eV})$. $(\mathrm{d} 1, \mathrm{~d} 2)$ No asymmetry but intrinsic SOC $(\lambda=0.16 \mathrm{eV})$ and Rashba SOC $(\gamma=0.16 \mathrm{eV})$. The red dashed lines in $(\mathrm{b} 2, \mathrm{c} 2, \mathrm{~d} 2)$ reproduce the absorptance spectrum (a2) of graphene, for comparison.

\section{S8.2 Results}

The results of Fig. S7(a1,a2) concerning graphene have already been the subject of much work[10, 11, 12, $9,13,14,15]$. The absorptance is equal to $\pi \alpha$ over a wide energy range, as long as the bands involved in the optical transitions keep a linear dispersion. The other figures show that the opening of a band gap, whether due to the asymmetry of the honeycomb sublattices [Fig. S7(b1,b2)] or to the intrinsic SOC [Fig. S7(c1,c2)], induces just above the optical threshold the appearance of a peak reflecting an absorptance considerably greater than $\pi \alpha$. This is attributed to the non-zero mass that appears at the edges of the bands, in the vicinity of the band gap [5]. When both charge carriers transform from massless to massive quasiparticles, the absorptance per valley is evolving as $\pi \alpha / 2 \rightarrow \pi \alpha$. This transformation, predicted in the case of $2 \mathrm{D}$ group-IV honeycomb crystals [16], is partial in the case where the massive character of the bands disappears very quickly as soon as we deviate in energy from the band edges. The same effect occurs in the case of PbSe quantum wells (Sect. VI of the main document).

When the spin degeneracy is lifted under the effect of Rashba SOC coupling, the peak reflecting the massive character splits into two peaks [Fig. S7(d1,d2)]. In any case, by moving towards the highest energies, 


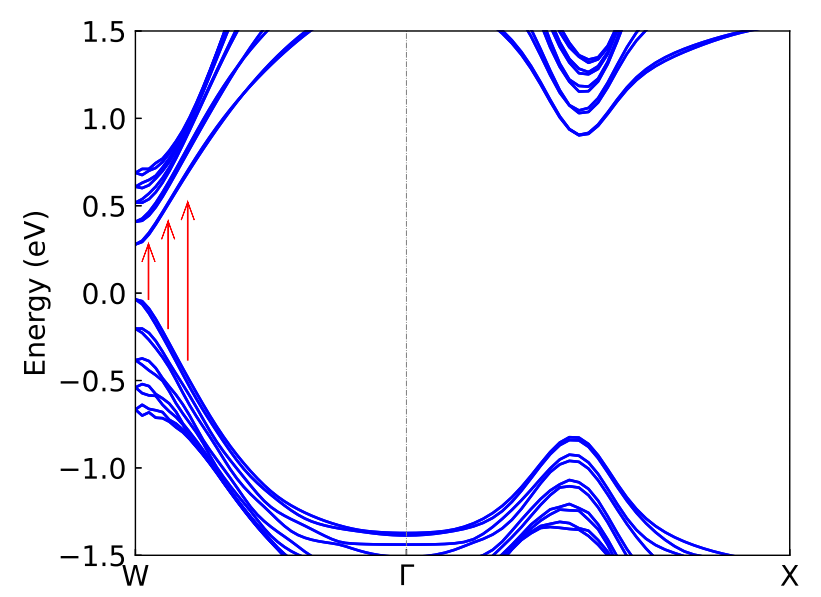

Figure S8: Lowest conduction subbands and highest valence subbands in a $6 \mathrm{~nm}$ quantum well of PbSe. The steps in the absorptance (Fig. 12 of the main document) are associated with the transitions indicated by red arrows.
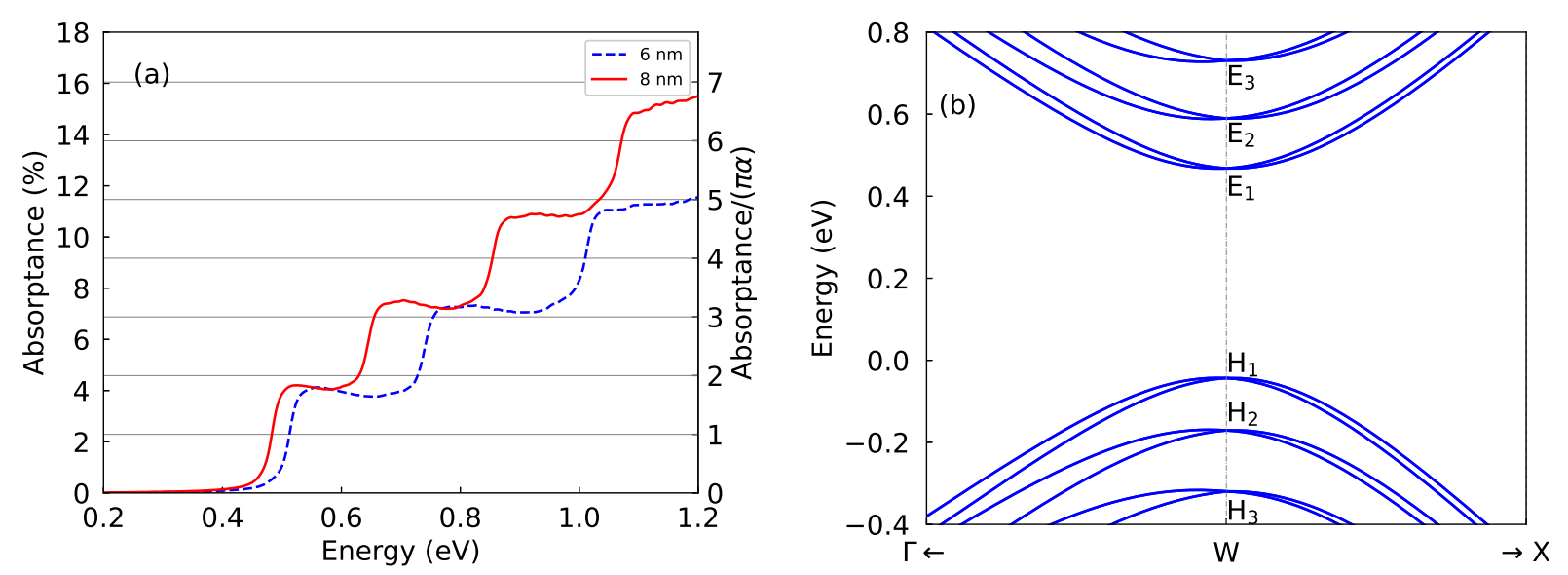

Figure S9: (a) Bare absorptance spectra calculated for (001) quantum wells of $\mathrm{PbS}$ (red solid line: thickness $=8 \mathrm{~nm}$; blue dashed line: $6 \mathrm{~nm}$ ). (b) Lowest conduction subbands and highest valence subbands in a $8 \mathrm{~nm}$ quantum well of $\mathrm{PbS}$. The steps in the absorptance are associated with the transitions $H_{i} \rightarrow E_{i}$.

the absorptance reaches the graphene reference value.

\section{S9 Additional results for quantum wells of rocksalt IV-VI semiconductors}

\section{S9.1 Band structure of the PbSe quantum well}

The band structure of the quantum well of PbSe discussed in the main document is presented in Fig. S8. 


\section{S9.2 Absorptance spectrum of a PbS quantum well}

The calculated band structure and absorptance spectra of $\mathrm{PbS}$ quantum wells are presented in Fig. S9. The first absorptance plateau is close to $2 \pi \alpha$, like in $\mathrm{PbSe}$ (main document). The height of the next plateaus is smaller than $2 \pi \alpha$, which could be due to the strongly anisotropic dispersion of higher subbands (Fig. S9b).

\section{References}

[1] Hui Fang, Hans A. Bechtel, Elena Plis, Michael C. Martin, Sanjay Krishna, Eli Yablonovitch, and Ali Javey. Quantum of optical absorption in two-dimensional semiconductors. Proc. Nat. Acad. Sci. USA, 110(29):11688-11691, 2013.

[2] E.O. Kane. Energy band structure in p-type germanium and silicon. J. Phys. Chem. Solids, 1(1):82-99, 1956.

[3] G. Fishman. Semi-conducteurs: les bases de la théorie K.P. Editions de l'Ecole Polytechnique, Palaiseau, 2010.

[4] G. Bastard. Wave mechanics applied to semiconductor heterostructures. Les Editions de Physique, Les Ulis, France, 1990.

[5] T. Stauber, D. Noriega-Pérez, and J. Schliemann. Universal absorption of two-dimensional systems. Phys. Rev. B, 91:115407, Mar 2015.

[6] P. R. Wallace. The band theory of graphite. Phys. Rev., 71:622-634, May 1947.

[7] A. H. Castro Neto, F. Guinea, N. M. R. Peres, K. S. Novoselov, and A. K. Geim. The electronic properties of graphene. Rev. Mod. Phys., 81:109-162, Jan 2009.

[8] C. L. Kane and E. J. Mele. Quantum spin hall effect in graphene. Phys. Rev. Lett., 95:226801, Nov 2005.

[9] R. R. Nair, P. Blake, A. N. Grigorenko, K. S. Novoselov, T. J. Booth, T. Stauber, N. M. R. Peres, and A. K. Geim. Fine structure constant defines visual transparency of graphene. Science, 320(5881):13081308, 2008.

[10] V. P. Gusynin, S. G. Sharapov, and J. P. Carbotte. Unusual microwave response of dirac quasiparticles in graphene. Phys. Rev. Lett., 96:256802, Jun 2006.

[11] N. M. R. Peres, F. Guinea, and A. H. Castro Neto. Electronic properties of disordered two-dimensional carbon. Phys. Rev. B, 73:125411, Mar 2006.

[12] L. A. Falkovsky and A. A. Varlamov. Space-time dispersion of graphene conductivity. Eur. Phys. J. B, 56(4):281-284, APR 2007.

[13] Kin Fai Mak, Matthew Y. Sfeir, Yang Wu, Chun Hung Lui, James A. Misewich, and Tony F. Heinz. Measurement of the optical conductivity of graphene. Phys. Rev. Lett., 101:196405, Nov 2008.

[14] Kin Fai Mak, Long Ju, Feng Wang, and Tony F. Heinz. Optical spectroscopy of graphene: From the far infrared to the ultraviolet. Solid State Commun., 152(15):1341 - 1349, 2012. 
[15] Thomas G. Pedersen. Analytic calculation of the optical properties of graphite. Phys. Rev. B, 67:113106, Mar 2003.

[16] Lars Matthes, Olivia Pulci, and Friedhelm Bechstedt. Massive dirac quasiparticles in the optical absorbance of graphene, silicene, germanene, and tinene. J. Phys. Condens. Matter, 25(39):395305, sep 2013. 